\title{
COMPOSITION OPERATORS ON VECTOR-VALUED HARMONIC FUNCTIONS AND CAUCHY TRANSFORMS
}

\author{
JUSSI LAITILA AND HANS-OLAV TYLLI
}

\begin{abstract}
Let $\varphi$ be an analytic self-map of the unit disk. The weak compactness of the composition operators $C_{\varphi}: f \mapsto f \circ \varphi$ is characterized on the vector-valued harmonic Hardy spaces $h^{1}(X)$, and on the spaces $C T(X)$ of vector-valued Cauchy transforms, for reflexive Banach spaces $X$. This provides a vector-valued analogue of results for composition operators which are due to Sarason, Shapiro and Sundberg, as well as Cima and Matheson. We also consider the operators $C_{\varphi}$ on certain spaces $w h^{1}(X)$ and $w C T(X)$ of weak type by extending an alternative approach due to Bonet, Domański and Lindström. Concrete examples based on minimal prerequisites highlight the differences between $h^{p}(X)$ (respectively, $C T(X))$ and the corresponding weak spaces.
\end{abstract}

\section{INTRODUCTION}

Let $\varphi$ be an analytic self-map of the unit disk $\mathbb{D}=\{z \in \mathbb{C}:|z|<1\}$. Properties of the composition operators

$$
C_{\varphi}: f \mapsto f \circ \varphi
$$

induced by $\varphi$ have been intensively studied on various Banach spaces of analytic functions defined on $\mathbb{D}$. The monographs $[\mathrm{CoM}]$ and [Sh2] contain the basic results related to e.g. the Hardy spaces $H^{p}$ and the (weighted) Bergman spaces. On the harmonic Hardy spaces $h^{p}$ the operators $C_{\varphi}$ were first considered by Sarason [S1].

The following omnibus result conveniently recapitulates several characterizations of and facts about the (weak) compactness of $C_{\varphi}$ on $h^{1}$ and $H^{1}$. Here $\mathcal{P} L^{1}$ denotes the image of $L^{1}(\mathbb{T})$ under the Poisson integral, so that $H^{1} \subset \mathcal{P} L^{1} \subset h^{1}$ as closed subspaces invariant under $C_{\varphi}$.

Omnibus Theorem. The following conditions are equivalent.

(1) $C_{\varphi}$ is (weakly) compact on $h^{1}$.

(2) $C_{\varphi}$ is (weakly) compact on $\mathcal{P} L^{1}$.

(3) $C_{\varphi}$ is (weakly) compact on $H^{1}$.

(4) $C_{\varphi}$ maps $h^{1}$ into $\mathcal{P} L^{1}$.

(5) $\int_{\mathbb{T}} \frac{1-|\varphi(\xi)|^{2}}{|\zeta-\varphi(\xi)|^{2}} d m(\xi)=\Re\left(\frac{\zeta+\varphi(0)}{\zeta-\varphi(0)}\right)$ for all $\zeta \in \mathbb{T}$.

Here $\varphi(\xi)=\lim _{r \rightarrow 1} \varphi(r \xi)$ is the a.e. radial limit function of $\varphi$ on $\mathbb{T}$.

2000 Mathematics Subject Classification. Primary: 47B33; Secondary: 46E40, 46G10.

The first author was supported by the Finnish Academy of Science and Letters (the Väisälä Foundation). Both authors were partly supported by the Academy of Finland project \#53893. 
The Omnibus Theorem combines the work of several authors. Sarason [S2] showed that the weak compactness of $C_{\varphi}$ on $H^{1}$ implies its compactness on $H^{1}$. Shapiro and Sundberg [ShS, p. 445] proved that a compact map $C_{\varphi}$ on $H^{1}$ is also compact on $h^{1}$. This means that (weak) compactness is equivalent for $C_{\varphi}$ between each of $h^{1}, \mathcal{P} L^{1}$ and $H^{1}$. The conditions (4) and (5) characterizing (weakly) compact maps $C_{\varphi}$ on $h^{1}$ were obtained by Sarason [S1, Prop. 2-4] (cf. [CM1, p. 61] for (5) in case $\varphi(0) \neq 0$ ). Above other concrete conditions on $\varphi$ could be used instead of (5), such as the earlier characterization by Shapiro [Sh1] of the compactness of $C_{\varphi}$ on $H^{2}$ in terms of the Nevanlinna counting function of $\varphi$ (recall here that the compactness of $C_{\varphi}$ on $H^{p}$ is independent of $0<p<\infty$ by a result of Shapiro and Taylor, see [CoM, Thm. 3.12]). Condition (5) was also reformulated by Sarason in terms of the absolute continuity of certain boundary type measures, cf. [CM1, p. 61] for a convenient description.

Bourdon and Cima $[\mathrm{BC}]$ observed that every composition operator $C_{\varphi}$ is bounded on $C T$, the Banach space consisting of the Cauchy transforms of Borel measures on the unit circle $\mathbb{T}$. Later Cima and Matheson [CM2] proved that if $C_{\varphi}$ is weakly compact on $C T$ then it is compact, and that the compactness of $C_{\varphi}$ on $C T$ is equivalent to its compactness on $H^{1}$.

Currently there is growing interest in the composition operators $C_{\varphi}$ in a vector-valued setting. For instance, the boundedness and the weak compactness of $f \mapsto f \circ \varphi$ have been studied on various spaces consisting of vectorvalued analytic functions $f: \mathbb{D} \rightarrow X$, such as the Hardy spaces $H^{p}(X)$ [LST], the (weighted) Bergman and Bloch spaces [LST], [BDL], and $B M O A(X)$ [L]. Here $X$ is a complex infinite-dimensional Banach space. The reference $[\mathrm{BF}]$ discusses a weighted locally convex setting, and other results related to vector-valued Hardy spaces are found in [HJ] and [SB].

The aim of this paper is to add to the picture from [LST], [BDL] and $[\mathrm{L}]$ by studying composition operators on the harmonic Hardy spaces $h^{p}(X)$ and the space $C T(X)$ of vector-valued Cauchy transforms. We establish a version of the Omnibus Theorem for $h^{1}(X)$, and of the results of [CM2] for $C T(X)$, by showing that $C_{\varphi}$ is weakly compact on $h^{1}(X)$ (respectively, on $C T(X)$ ) if and only if condition (5) holds and $X$ is reflexive. (Note that $C_{\varphi}$ is never compact on these vector-valued spaces once $X$ is infinite-dimensional, since $C_{\varphi}$ preserves the constant functions $f_{x}(z) \equiv x$ for $x \in X$.)

A novel feature in the vector-valued setting is that there usually are more than one canonical $X$-valued space which correspond to a given classical scalar-valued space. In section 5 we characterize the weakly compact composition operators on a class $w E(X)$ of weak spaces of vector-valued harmonic functions. This complements analogous results by Bonet, Domański and Lindström [BDL] for weak spaces of analytic functions. Our result applies for instance to the weak harmonic Hardy space $w h^{1}(X)$ considered by e.g. Blasco [B1] (a harmonic function $f: \mathbb{D} \rightarrow X$ belongs to $w h^{1}(X)$ if $x^{*} \circ f \in h^{1}$ for $\left.x^{*} \in X^{*}\right)$. The approach for $w E(X)$ is different from that for spaces such as $h^{1}(X)$ or $C T(X)$.

The final section exhibits, for any infinite-dimensional $X$ and $1 \leq p<\infty$, concrete functions $f: D \rightarrow X$ for which $f \in w h^{p}(X) \backslash h^{p}(X)$. The analogous question is also addressed for the case $C T(X)$, where the results are less 
complete. Our examples are motivated by the two competing approaches above, and they simplify and complement earlier ones due to Blasco [B1], Freniche, Garcia-Vazguez and Rodriguez-Piazza [FGR1], [FGR2], and the first-named author [L].

\section{Preliminaries}

In the sequel $X=\left(X,\|\cdot\|_{X}\right)$ will always be a complex Banach space. For $1 \leq p<\infty$ let $L^{p}(X) \equiv L^{p}(\mathbb{T}, X)$ denote the space of Bochner-integrable functions $g: \mathbb{T} \rightarrow X$ satisfying

$$
\|g\|_{L^{p}(X)}=\left(\int_{\mathbb{T}}\|g\|_{X}^{p} d m\right)^{1 / p}<\infty
$$

where $m$ is the normalized Lebesgue measure on $\mathbb{T}$.

Recall that $f: \mathbb{D} \rightarrow X$ is a harmonic function if $x^{*} \circ f: \mathbb{D} \rightarrow \mathbb{C}$ is harmonic for every $x^{*} \in X^{*}$ (that is, $f$ is weakly harmonic). We note that $f: \mathbb{D} \rightarrow X$ is harmonic if and only if there is a sequence $\left(a_{n}\right) \subset X$ so that

$$
f(z)=\sum_{n=0}^{\infty} a_{n} z^{n}+\sum_{n=1}^{\infty} a_{-n} \bar{z}^{n}, \quad z \in \mathbb{D},
$$

where the series converges absolutely and uniformly on compact subsets of $\mathbb{D}$ (see e.g. [H1, p. 13] or [H2, p. 352] for both these facts). Define $f_{r}: \mathbb{T} \rightarrow X$ by $f_{r}(\xi)=f(r \xi)$ for maps $f: \mathbb{D} \rightarrow X$ and $0<r<1$. The harmonic function $f: \mathbb{D} \rightarrow X$ belongs to the harmonic Hardy space $h^{p}(X)=h^{p}(\mathbb{D}, X)$ for $1 \leq p<\infty$ if

$$
\|f\|_{h^{p}(X)}=\sup _{0<r<1}\left\|f_{r}\right\|_{L^{p}(X)}<\infty .
$$

The analytic Hardy space $H^{p}(X)=H^{p}(\mathbb{D}, X)$ is the closed subspace of $h^{p}(X)$ consisting of the analytic functions $f: \mathbb{D} \rightarrow X$ for $1 \leq p<\infty$.

Let $\Sigma(\mathbb{T})$ be the Borel $\sigma$-algebra on the unit circle $\mathbb{T}$, and $M(X)=$ $M(\mathbb{T}, X)$ be the Banach space of the countably additive vector measures $\mu: \Sigma(\mathbb{T}) \rightarrow X$ equipped with the total variation norm $\|\mu\|_{M(X)}=|\mu|(\mathbb{T})$. Here $|\mu|$ is the variation of $\mu$. Let $\mathcal{P}[\mu]: \mathbb{D} \rightarrow X$ be the function defined by the Poisson integral

$$
\mathcal{P}[\mu](z)=\int_{\mathbb{T}} \frac{1-|z|^{2}}{|\zeta-z|^{2}} d \mu(\zeta), \quad z \in \mathbb{D} .
$$

The integral $\int_{\mathbb{T}} g d \mu$ of a continuous map $g: \mathbb{T} \rightarrow \mathbb{C}$ with respect to the vector measure $\mu \in M(X)$, as in (2.2), is defined via approximation by simple functions, see [DU, pp. 5-6]. The easy estimate $\left\|\int_{\mathbb{T}} g d \mu\right\|_{X} \leq \int_{\mathbb{T}}|g| d|\mu|$ will be used subsequently. It is not difficult to check that $\mathcal{P}[\mu]$ admits a harmonic power series representation (2.1), where $a_{n}=\widehat{\mu}_{n}=\int_{\mathbb{T}} \bar{\zeta}^{n} d \mu(\zeta)$ for $n \in \mathbb{Z}$. The coefficients satisfy $\left\|\widehat{\mu}_{n}\right\|_{X} \leq\|\mu\|_{M(X)}$ for $n \in \mathbb{Z}$.

The following fundamental vector-valued result is less well-known. (The case $X=\mathbb{C}$ is a classical fact, see e.g. [R, Thm. 11.30].)

Theorem 2.1. Let $X$ be an arbitrary complex Banach space. Then the map $\mu \mapsto \mathcal{P}[\mu]$ is an isometric isomorphism from $M(X)$ onto $h^{1}(X)$. 
The preceding theorem is due in varying degrees of generality to Ryan, Grossetête, Heins and Hensgen. An argument is sketched e.g. on [H3, p. 90] and full details are found in [H1, Satz 1.5] (see also [B2, Thm. 1] for a related context).

For simplicity we will put $h^{1}=h^{1}(\mathbb{C}), L^{1}=L^{1}(\mathbb{C}), H^{1}=H^{1}(\mathbb{C})$ etc. in the scalar-valued case $X=\mathbb{C}$. We refer the reader to e.g. [D], [R] for further basic facts about the analytic Hardy spaces, and to e.g. [B1], [H1] or [H2] for vector-valued spaces of harmonic functions.

\section{COMPosition OPERATORS ON HARMOniC HARDY SPACES}

It follows from Littlewood's subordination principle that any $C_{\varphi}$ is bounded on $h^{p}$ for $1 \leq p<\infty$, see [S1, Prop. 1] or [ShS, p. 444]. The map $C_{\varphi}$ also acts boundedly on the subspace $\mathcal{P} L^{1}$, since it preserves the closure of the harmonic polynomials in $h^{1}$. Similarly, $C_{\varphi}$ preserves the analytic Hardy spaces $H^{p}$.

In this section we extend the Omnibus Theorem by characterizing the weakly compact composition operators on $h^{1}(X)$ for reflexive spaces $X$. Let $X$ be a complex Banach space. We isometrically identify $L^{1}(X)$ with the closed subspace $\left\{g d m: g \in L^{1}(X)\right\} \subset M(X)$, where $\mu=g d m \in M(X)$ is defined by $\mu(E)=\int_{E} g(\zeta) d m(\zeta)$ for $E \in \Sigma(\mathbb{T})$. We denote by $\mathcal{P} L^{1}(X)$ the image of $L^{1}(X)$ under the Poisson integral. It is not difficult to check that $\mathcal{P} L^{1}(X)$ is the closed subspace of $h^{1}(X)$ spanned by the $X$-valued harmonic polynomials $\sum_{k=0}^{m} x_{k} z^{k}+\sum_{k=1}^{n} x_{-k} \bar{z}^{k}(m, n \in \mathbb{N})$, see e.g. [H2, p. 352] and Theorem 2.1.

The vector-valued setting appears more delicate initially, in part because of the possible influence of various Radon-Nikodým type properties. We next recall these facts. For any $X$ the radial limit function $f(\xi)=\lim _{r \rightarrow \infty} \mathcal{P}[f](r \xi)$ exists in the norm of $X$ a.e. on $\mathbb{T}$ for $f \in L^{1}(X)$, see e.g. [Bu, Thm. 2.5], [H1, Cor. 1.9] or [H2, p. 353]. By contrast, every $f \in h^{1}(X)$ admits a radial limit function $\lim _{r \rightarrow \infty} f(r \xi)$ a.e. on $\mathbb{T}$ if and only if $X$ has the Radon-Nikodým property (RNP), see [B3, Thm. 2.2], [H1, Satz 1.12] or [H3, Thm. 2.1]. It is also relevant to consider the subspace $H^{1}(X) \cap \mathcal{P} L^{1}(X) \subset h^{1}(X)$. Here

$$
H^{1}(X) \cap \mathcal{P} L^{1}(X)=\mathcal{P} L_{a}^{1}(X),
$$

where $L_{a}^{1}(X)=\left\{f \in L^{1}(X): \widehat{f}_{n}=0, n<0\right\}$. In fact, $\mathcal{P} L_{a}^{1}(X)$ is the closed subspace of $H^{1}(X)$ consisting of the functions which admit radial limits a.e. on $\mathbb{T}$, see e.g. [H1, Satz 2.7] or [H2, p. 355]. Moreover, $\mathcal{P} L_{a}^{1}(X)=H^{1}(X)$ if and only if $X$ has the analytic Radon-Nikodým property (ARNP), see [Bu, p. 1055] or [BD, p. 105]. Hence $H^{1}(X)$ and $\mathcal{P} L^{1}(X)$ are not always comparable spaces. We refer to e.g. [Bu], [BD], [B3] or [H3] for the relevant discussion of the (A)RNP (these properties will not be used explicitly here). Analogous facts hold for $1<p<\infty$, but we will not pursue this case here.

The potential absence of radial limits for $f \in h^{p}(X)$ is a technical complication for composition operators on these spaces, which has to be circumvented in the arguments. Nevertheless, it is a basic fact that every map $C_{\varphi}$ is bounded on $h^{p}(X)$ for $1 \leq p<\infty$ and any $X$. We recall for convenience an explicit argument, which modifies a well-known scalar proof (cf. [D, p. 29]). The case $H^{p}(X)$ was treated somewhat differently in [LST, p. 298]. 
Proposition 3.1. Let $X$ be a complex Banach space, and $\varphi: \mathbb{D} \rightarrow \mathbb{D}$ be an analytic map. Then $C_{\varphi}$ is bounded $h^{p}(X) \rightarrow h^{p}(X)$ for $1 \leq p<\infty$, and

$$
\left\|C_{\varphi}\right\| \leq\left(\frac{1+|\varphi(0)|}{1-|\varphi(0)|}\right)^{1 / p} .
$$

The closed subspaces $\mathcal{P} L^{1}(X), H^{1}(X)$ and $\mathcal{P} L_{a}^{1}(X)$ of $h^{1}(X)$ are invariant under $C_{\varphi}$.

If $f: \mathbb{D} \rightarrow X$ is a harmonic function then the map $v_{f}: z \mapsto\|f(z)\|_{X}^{p}$ is subharmonic on $\mathbb{D}$, so that $\|f\|_{h^{p}(X)}=\lim _{r \rightarrow 1}\left\|f_{r}\right\|_{L^{p}(X)}$. The following facts can be checked just as in the scalar case (cf. [D, Thm. 2.12] or [H3, p. 92]): A harmonic map $f: \mathbb{D} \rightarrow X$ belongs to $h^{p}(X)$ if and only if $v_{f}$ has a harmonic majorant on $\mathbb{D}$. Moreover, for $f \in h^{p}(X)$ the least harmonic majorant $h_{f}$ of $v_{f}$ is given by

$$
h_{f}(z)=\lim _{r \rightarrow 1} \mathcal{P}\left[\left\|f_{r}\right\|_{X}^{p}\right](z), \quad z \in \mathbb{D} .
$$

Proof of Proposition 3.1. Let $f \in h^{p}(X)$. Then $h_{f} \circ \varphi$ is a harmonic majorant on $\mathbb{D}$ of the subharmonic map $v_{f \circ \varphi}$. The mean value property yields that

$$
\begin{aligned}
\int_{\mathbb{T}}\|(f \circ \varphi)(r \zeta)\|_{X}^{p} d m(\zeta) & \leq \int_{\mathbb{T}}\left(h_{f} \circ \varphi\right)(r \zeta) d m(\zeta) \\
& =h_{f}(\varphi(0))=\lim _{r \rightarrow 1} \mathcal{P}\left[\left\|f_{r}\right\|_{X}^{p}\right](\varphi(0)) .
\end{aligned}
$$

Hence

$$
\lim _{r \rightarrow 1}\left\|(f \circ \varphi)_{r}\right\|_{L^{p}(X)}^{p} \leq \lim _{r \rightarrow 1} \mathcal{P}\left[\left\|f_{r}\right\|_{X}^{p}\right](\varphi(0)) \leq \frac{1+|\varphi(0)|}{1-|\varphi(0)|}\|f\|_{h^{p}(X)},
$$

since $\frac{1-|z|^{2}}{|\zeta-z|^{2}} \leq \frac{1+|z|}{1-|z|}$ for $\zeta \in \mathbb{T}$.

Clearly $C_{\varphi}$ maps the $X$-valued harmonic polynomials into the closure $\mathcal{P} L^{1}(X)$ of the harmonic polynomials in $h^{1}(X)$, and preserves the analyticity. Thus $\mathcal{P} L_{a}^{1}(X)=\mathcal{P} L^{1}(X) \cap H^{1}(X)$ is also invariant under $C_{\varphi}$.

The main result of this section extends the Omnibus Theorem to the vector-valued setting. Note that if $C_{\varphi}$ is weakly compact $h^{1}(X) \rightarrow h^{1}(X)$, then the closed unit ball $B_{X}=\{x \in X:\|x\| \leq 1\}$ is weakly compact in $X$, since $C_{\varphi}\left(f_{x}\right)=f_{x}$ for the constants $f_{x}(z) \equiv x$ (where $\left.x \in X\right)$. This means that $X$ is reflexive. Recall that $H^{1}(X)=\mathcal{P} L_{a}^{1}(X)$ for reflexive $X$, because such spaces have the (A)RNP, see [DU, Thm. III.1.6]. Thus the space $\mathcal{P} L_{a}^{1}(X)$ is also implicit in the conditions below. The argument below also provides an alternative approach to [LST, Thm. 3.(ii)], where it was shown that $C_{\varphi}$ is weakly compact on $H^{1}(X)$ if and only if $\varphi$ satisfies Shapiro's condition and $X$ is reflexive.

Theorem 3.2. Let $X$ be a reflexive Banach space and $\varphi$ be an analytic self-map of $\mathbb{D}$. Then the following conditions are equivalent.

(1) $C_{\varphi}$ is weakly compact on $h^{1}(X)$.

(2) $C_{\varphi}$ is weakly compact on $\mathcal{P} L^{1}(X)$.

(3) $C_{\varphi}$ is weakly compact on $H^{1}(X)$.

(4) $C_{\varphi}$ maps $h^{1}(X)$ into $\mathcal{P} L^{1}(X)$.

(5) $\int_{\mathbb{T}} \frac{1-|\varphi(\xi)|^{2}}{|\zeta-\varphi(\xi)|^{2}} d m(\xi)=\Re\left(\frac{\zeta+\varphi(0)}{\zeta-\varphi(0)}\right)$ for all $\zeta \in \mathbb{T}$. 
Here $\varphi$ denotes the a.e. radial limit function on $\mathbb{T}$.

Proof. It is convenient to use the non-trivial fact that $H^{1}(X) \subset \mathcal{P} L^{1}(X)$ if $X$ is reflexive (see above). It is then obvious that $(1) \Rightarrow(2) \Rightarrow(3)$, since $H^{1}(X)$ and $\mathcal{P} L^{1}(X)$ are invariant subspaces for $C_{\varphi}$ in $h^{1}(X)$. (The alternative is to verify separately that $(1) \Rightarrow(2) \Rightarrow(5)$ and $(1) \Rightarrow(3) \Rightarrow(5)$ by slightly modifying the ideas below.)

$(3) \Rightarrow(5)$. Fix $x \in X$ and $x^{*} \in X^{*}$ so that $x^{*}(x)=1$, and define the bounded operators $A: H^{1} \rightarrow H^{1}(X), B: H^{1}(X) \rightarrow H^{1}$ by $A f=x f$ and $B g=x^{*} \circ g$ for $f \in H^{1}$ and $g \in H^{1}(X)$. It follows that

$$
B \circ C_{\varphi} \circ A=\tilde{C}_{\varphi},
$$

where $\tilde{C}_{\varphi}$ denotes the corresponding composition map on $H^{1}$. If $C_{\varphi}$ is weakly compact on $H^{1}(X)$, then $\tilde{C}_{\varphi}$ is weakly compact on $H^{1}$, and (5) follows by appealing to the Omnibus Theorem.

$(4) \Rightarrow(5)$. The factorization (3.2) remains valid for $h^{1}$ and $h^{1}(X)$, where $B$ maps $\mathcal{P} L^{1}(X)$ into $\mathcal{P} L^{1}$ since $x^{*} \circ \mathcal{P}[g]=\mathcal{P}\left[x^{*} \circ g\right]$ for $g \in L^{1}(X)$. If $C_{\varphi}$ maps $h^{1}(X)$ into $\mathcal{P} L^{1}(X)$, then $\tilde{C}_{\varphi}$ maps $h^{1}$ into $\mathcal{P} L^{1}$ by (3.2) and we may again apply the Omnibus Theorem.

The argument for the main implications $(5) \Rightarrow(1)$ and $(5) \Rightarrow(4)$ will be based on a suitable vector-valued variant of the ideas of Sarason [S1, Prop. 2 and 4]. We first state a simple auxiliary lemma.

Lemma 3.3. Let $X$ be a reflexive Banach space and $\varphi$ be an analytic selfmap of $\mathbb{D}$ satisfying $\|\varphi\|_{\infty} \equiv \sup |\varphi(z)|<1$. Then $C_{\varphi}$ is weakly compact $h^{1}(X) \rightarrow h^{1}(X)$.

Proof. It suffices to check that the operators $C_{r}: f \mapsto f(r \cdot)$ are weakly compact on $h^{1}(X)$ for $0<r<1$, since $C_{\varphi}=C_{\varphi / r} C_{r}$ for $\|\varphi\|_{\infty}<r<$ 1. We will approximate $C_{r}$ using the bounded truncation operators $P_{n}$ on $h^{1}(X)$, where $P_{n} f\left(s e^{i \theta}\right)=\sum_{k=-n}^{n} \widehat{\mu}_{k} s^{|k|} e^{i k \theta}$ for $f\left(s e^{i \theta}\right)=\sum_{k=-\infty}^{\infty} \widehat{\mu}_{k} s^{|k|} e^{i k \theta}$ in $h^{1}(X)$ and $n \geq 0$. Here $f=\mathcal{P}[\mu]$ for some $\mu \in M(X)$, cf. Theorem 2.1.

Let $\varepsilon>0$ and fix $n_{0}$ so that $2 \sum_{k=n_{0}+1}^{\infty} r^{n}<\varepsilon$. For any $z=s e^{i \theta} \in \mathbb{D}$ and $f \in h^{1}(X)$ with $f\left(s e^{i \theta}\right)=\sum_{k=-\infty}^{\infty} \widehat{\mu}_{k} s^{|k|} e^{i k \theta}$ we get that

$$
\begin{aligned}
\left\|\left(C_{r}-P_{n_{0}} C_{r}\right) f(z)\right\|_{X} & \leq \sum_{k=n_{0}+1}^{\infty}\left\|\widehat{\mu}_{k}\right\|_{X} s^{k} r^{k}+\sum_{k=-\infty}^{-\left(n_{0}+1\right)}\left\|\widehat{\mu}_{k}\right\|_{X} s^{|k|} r^{|k|} \\
& \leq 2\|f\|_{h^{1}(X)} \sum_{k=n_{0}+1}^{\infty} r^{n} \leq \varepsilon\|f\|_{h^{1}(X)},
\end{aligned}
$$

because $\left\|\widehat{\mu}_{k}\right\|_{X} \leq\|\mu\|_{M(X)}=\|f\|_{h^{1}(X)}$ for $k \in \mathbb{Z}$ by Theorem 2.1. Hence $\left\|\left(C_{r}-P_{n_{0}} C_{r}\right) f\right\|_{h^{1}(X)} \leq \varepsilon\|f\|_{h^{1}(X)}$, so that $\left\|C_{r}-P_{n} C_{r}\right\| \rightarrow 0$ as $n \rightarrow \infty$. The proof is completed by noting that every $P_{n}$ is weakly compact on $h^{1}(X)$, since $P_{n}$ factors through the reflexive direct sum $\ell_{2}^{2 n+1}(X)$ (cf. the proof of [LST, Prop. 2]).

Proof of (5) $\Rightarrow$ (1) and (5) $\Rightarrow$ (4). The operators $C_{r \varphi}=C_{\varphi} C_{r}$ are weakly compact on $h^{1}(X)$ for $0<r<1$ by Lemma 3.3. Moreover, $C_{r \varphi}$ also maps $h^{1}(X)$ into $\mathcal{P} L^{1}(X)$, since $C_{r}(f)$ admits a continuous extension to $\overline{\mathbb{D}}$ for 
$f \in h^{1}(X)$ and $C_{\varphi}\left(\mathcal{P} L^{1}(X)\right) \subset \mathcal{P} L^{1}(X)$. We may assume that $\varphi(0)=0$ by composing $\varphi$ with a suitable Möbius transformation $\psi$ of $\mathbb{D}$, since $C_{\psi}$ is a linear isomorphism of $h^{1}(X)$ that preserves $\mathcal{P} L^{1}(X)$. In the case where $\varphi(0)=0$ condition (5) becomes

$$
\int_{\mathbb{T}} \frac{1-|\varphi(\xi)|^{2}}{|\zeta-\varphi(\xi)|^{2}} d m(\xi)=1, \quad \zeta \in \mathbb{T}
$$

We first observe that to deduce conditions (1) and (4) from (3.3) it will suffice to show that for every $\varepsilon>0$ there is $r_{0} \in(0,1)$ so that

$$
\left\|\left(C_{r \varphi}-C_{s \varphi}\right) f\right\|_{h^{1}(X)} \leq \varepsilon\|f\|_{h^{1}(X)}
$$

holds for all $s, r \geq r_{0}$ and $f \in h^{1}(X)$. Indeed, (3.4) implies that $C_{r \varphi}$ converges in the uniform norm to some weakly compact operator $S$ on $h^{1}(X)$ as $r \rightarrow 1$. One must have $S=C_{\varphi}$. In fact, convergence in $\|\cdot\|_{h^{1}(X)}$ implies uniform convergence on the compact subsets of $\mathbb{D}$ (recall that $\|\mathcal{P}[\mu](z)\|_{X} \leq$ $2(1-|z|)^{-1}\|\mathcal{P}[\mu]\|_{h^{1}(X)}$ for $z \in \mathbb{D}$ by Theorem 2.1$)$, while by continuity $C_{r \varphi} f \rightarrow C_{\varphi} f$ uniformly on the compact subsets of $\mathbb{D}$ as $r \rightarrow 1$ for $f \in h^{1}(X)$.

Define the kernel functions $K_{r}$ on $\mathbb{T} \times \mathbb{T}$ for $0<r<1$ by

$$
K_{r}(\xi, \zeta)=\frac{1-|r \varphi(\xi)|^{2}}{|\zeta-r \varphi(\xi)|^{2}}
$$

Here $\varphi$ is the a.e. radial limit function on $\mathbb{T}$, so that $K_{r}$ is defined a.e. in $\xi$, while the maps $\zeta \mapsto K_{r}(\xi, \zeta)$ are continuous. We recall next from [S1, Prop. 2] (cf. also [CM1, p. 61]) how to deduce from (3.3) that for every $\varepsilon>0$ there is $r_{0} \in(0,1)$ so that

$$
\sup _{\zeta \in \mathbb{T}} \int_{\mathbb{T}}\left|K_{r}(\xi, \zeta)-K_{s}(\xi, \zeta)\right| d m(\xi)<\varepsilon
$$

for all $r, s \geq r_{0}$. The crux is to verify that

$$
\left\|K_{r}(\cdot, \zeta)-\frac{1-|\varphi(\cdot)|^{2}}{|\zeta-\varphi(\cdot)|^{2}}\right\|_{L^{1}} \rightarrow 1 \quad \text { as } r \rightarrow 1
$$

for $\zeta \in \mathbb{T}$. Fix $\zeta \in \mathbb{T}$ and recall that $\left\|K_{r}(\cdot, \zeta)\right\|_{L^{1}}=1$ for $0<r<1$ (see $\left[\right.$ S1, Lemma 3]). Hence $\left\|K_{r}(\cdot, \zeta)\right\|_{L^{1}} \rightarrow \int_{\mathbb{T}} \frac{1-|\varphi(\xi)|^{2}}{|\zeta-\varphi(\xi)|^{2}} d m(\xi)$ as $r \rightarrow 1$ by (3.3), while $K_{r}(\xi, \zeta) \rightarrow \frac{1-|\varphi(\xi)|^{2}}{|\zeta-\varphi(\xi)|^{2}}$ a.e. in $\xi \in \mathbb{T}$ as $r \rightarrow 1$. Thus (3.6) follows from a classical fact due to Dunford and Pettis (cf. [DS, III.3.6]). In particular, $\left\|K_{r}(\cdot, \zeta)-K_{s}(\cdot, \zeta)\right\|_{L^{1}} \rightarrow 0$ as $r, s \rightarrow 1$ for each $\zeta \in \mathbb{T}$, which implies that (3.5) holds.

To continue the argument let $f=\mathcal{P}[\mu] \in h^{1}(X)$ be arbitrary and take $r, s \geq r_{0}$. Then $f \circ(r \varphi)-f \circ(s \varphi) \in \mathcal{P} L^{1}(X)$ and

$$
\begin{aligned}
\|(\mathcal{P}[\mu])(r \varphi(\xi))-(\mathcal{P}[\mu])(s \varphi(\xi))\|_{X} & =\left\|\int_{\mathbb{T}}\left(K_{r}(\xi, \zeta)-K_{s}(\xi, \zeta)\right) d \mu(\zeta)\right\|_{X} \\
& \leq \int_{\mathbb{T}}\left|K_{r}(\xi, \zeta)-K_{s}(\xi, \zeta)\right| d|\mu|(\zeta),
\end{aligned}
$$


for a.e. $\xi \in \mathbb{T}$. Since the map $f \circ(r \varphi)-f \circ(s \varphi)$ only involves the values of $f$ on compact subsets of $\mathbb{D}$ one gets from (3.5) and Fubini's theorem that

$$
\begin{aligned}
\|f \circ(r \varphi)-f \circ(s \varphi)\|_{h^{1}(X)} & =\int_{\mathbb{T}}\|f(r \varphi(\xi))-f(s \varphi(\xi))\|_{X} d m(\xi) \\
& \leq \int_{\mathbb{T}} \int_{\mathbb{T}}\left|K_{r}(\xi, \zeta)-K_{s}(\xi, \zeta)\right| d m(\xi) d|\mu|(\zeta) \\
& \leq \varepsilon\|f\|_{h^{1}(X)}
\end{aligned}
$$

for $r, s \geq r_{0}$. Thus (3.4) follows from (3.5), and the proof is complete.

Remarks. (i) For the implications $(5) \Rightarrow(1)$ and $(5) \Rightarrow(4)$ it is also possible to apply the factorization $C_{\varphi_{r}}=C_{r} C_{\varphi}$ and the related continuous kernel

functions $\tilde{K}_{r}(\xi, \zeta)=\frac{1-|\varphi(r \xi)|^{2}}{|\zeta-\varphi(r \xi)|^{2}},(\xi, \zeta) \in \mathbb{T} \times \mathbb{T}$ and $0<r<1$. The details of this variation are left to the reader.

(ii) $h^{p}(X)$ is a reflexive space if $X$ is reflexive and $1<p<\infty$ (cf. [BD, Thm. 2 and Prop. 3], [H2, p. 353] and [DU, Cor. IV.1.2]), whence any operator on $h^{p}(X)$ is weakly compact. This is our reason for not pursuing the case $1<p<\infty$ more closely.

(iii) The following general variant of Lemma 3.3 holds with a similar proof for closed operator ideals $I$ in the sense of Pietsch $[\mathrm{P}]:$ if $\|\varphi\|_{\infty}<1$ and the identity map $I_{X} \in I(X)$, then $C_{\varphi} \in I\left(h^{1}(X)\right)$.

This fact leads to different vector-valued extensions of the Omnibus Theorem. For instance, recall that $S: X \rightarrow X$ is a weakly conditionally compact operator if every sequence $\left(x_{n}\right) \subset B_{X}$ admits a subsequence $\left(x_{n_{k}}\right)$ so that $\left(S x_{n_{k}}\right)$ is weakly Cauchy. By Rosenthal's $\ell^{1}$-theorem one has that $I_{X}$ is not weakly conditionally compact if and only if $X$ contains a linearly isomorphic copy of $\ell^{1}$, see [LT, 2.e.5]. By suitable modifications in the proof of Theorem 3.2 one obtains a version concerning weakly conditionally compact compositions $C_{\varphi}$ on $h^{1}(X)$, where $X$ does not contain any isomorphic copies of $\ell^{1}$. This class contains spaces $X$ that fail to have the ARNP (such as $c_{0}$ ). One may also add the equivalent condition that $C_{\varphi}$ is weakly conditionally compact $\mathcal{P} L_{a}^{1}(X) \rightarrow \mathcal{P} L_{a}^{1}(X)$. We leave the details to the reader (see also [LST, Thm. 7]).

\section{Composition operators on vector-valued Cauchy TRANSFORMS}

As an application of the preceding section we discuss here composition operators on the space $C T(X)$ of $X$-valued Cauchy transforms, and we extend some basic results of Bourdon, Cima and Matheson [BC],[CM2] from the scalar-valued case. We begin by recalling the setting.

The Cauchy transform $\mathcal{C}[\mu]$ of the scalar measure $\mu \in M$ is the analytic function $\mathbb{D} \rightarrow \mathbb{C}$ given by

$$
\mathcal{C}[\mu](z)=\int_{\mathbb{T}} \frac{1}{1-\bar{\zeta} z} d \mu(\zeta)=\sum_{n=0}^{\infty} \widehat{\mu}_{n} z^{n}, \quad z \in \mathbb{D} .
$$

Let $X$ be a complex Banach space. If $\mu \in M(X)$ is a vector measure, then (4.1) defines an analytic function $\mathcal{C}[\mu]: \mathbb{D} \rightarrow X$. The vector-valued extension $C T(X)$ of the Banach space $C T$ is introduced by analogy with the 
scalar-valued case as follows: $C T(X)$ is the linear space $\{\mathcal{C}[\mu]: \mu \in M(X)\}$ equipped with the norm

$$
\|\mathcal{C}[\mu]\|_{C T(X)}=\inf \left\{\|\lambda\|_{M(X)}: \mathcal{C}[\mu]=\mathcal{C}[\lambda]\right\} .
$$

Note that $\mathcal{C}[\mu]=\mathcal{C}[\lambda]$ if and only if $\mu=\lambda+\nu$ for some $\nu \in M(X)$ satisfying $\widehat{\nu}_{n}=0$ for $n \geq 0$. Hence

$$
\|\mathcal{C}[\mu]\|_{C T(X)}=\inf \left\{\|\mu+\nu\|_{M(X)}: \nu \in \overline{M_{a, 0}}(X)\right\},
$$

where $\overline{M_{a, 0}}(X)=\left\{\nu \in M(X): \widehat{\nu}_{n}=0\right.$ for $\left.n \geq 0\right\}$, so that $C T(X)$ is isometric to the quotient space $M(X) / \overline{M_{a, 0}}(X)$. Since the Poisson integral $\mu \mapsto \mathcal{P}[\mu]$ is an isometry from $M(X)$ onto $h^{1}(X)$ for any Banach space $X$ by Theorem 2.1 , we may also isometrically identify

$$
C T(X)=h^{1}(X) / \overline{H_{0}^{1}}(X),
$$

where $\overline{H_{0}^{1}}(X)=\mathcal{P}\left(\overline{M_{a, 0}}(X)\right)$. Note that here

$$
\overline{H_{0}^{1}}(X)=\left\{f \in h^{1}(X): f \text { is anti-analytic and } f(0)=0\right\},
$$

see e.g. [H1, Lemma 2.2]. The map $\pi: h^{1}(X) \rightarrow C T(X)$ defined by

$$
\pi(\mathcal{P}[\mu])=\mathcal{C}[\mu], \quad \mu \in M(X),
$$

satisfies $B_{C T(X)}=\overline{\pi\left(B_{h^{1}(X)}\right)}$, because it is essentially the quotient map $M(X) \rightarrow C T(X)$. Here $B_{C T(X)}=\left\{f \in C T(X):\|f\|_{C T(X)} \leq 1\right\}$ is the closed unit ball. We refer to the recent survey [CMR] for more information about the Cauchy transform and the space $C T$.

It was shown in [BC, Thm. 4.3] that the composition operator $C_{\varphi}$ is bounded on $C T$ for any analytic self-map $\varphi$ of $\mathbb{D}$. Later Cima and Matheson [CM2, Thm. 1] established the (sharp) upper bound

$$
\left\|C_{\varphi}\right\| \leq \frac{1+2|\varphi(0)|}{1-|\varphi(0)|} .
$$

To study the maps $C_{\varphi}$ on $C T(X)$ it is convenient to introduce a related composition-type operator $S_{\varphi}$ on $M(X)$ by mimicking Sarason [S1]. (In fact, $S_{\varphi}$ was the main object of study in [S1].) Let $\mu \in M(X)$. Since $\mathcal{P}$ is an isometric isomorphism $M(X) \rightarrow h^{1}(X)$ by Theorem 2.1 and $\mathcal{P}[\mu] \circ \varphi \in h^{1}(X)$ by Proposition 3.1, there is a unique $\nu \in M(X)$ so that $\mathcal{P}[\mu] \circ \varphi=\mathcal{P}[\nu]$. Thus $\mu \mapsto \nu$ defines a linear operator $S_{\varphi}: M(X) \rightarrow M(X)$ satisfying

$$
\mathcal{P}\left[S_{\varphi}(\mu)\right]=\mathcal{P}[\mu] \circ \varphi, \quad \mu \in M(X) .
$$

In other words, $S_{\varphi}=\mathcal{P}^{-1} \circ C_{\varphi} \circ \mathcal{P}$, where $C_{\varphi}$ is the corresponding composition operator on $h^{1}(X)$.

Theorem 4.1. Let $X$ be a complex Banach space and $\varphi$ be an analytic selfmap of $\mathbb{D}$. Then $C_{\varphi}$ is bounded $C T(X) \rightarrow C T(X)$ and the estimate (4.4) holds on $C T(X)$.

The argument is based on the following vector-valued modification of $[\mathrm{CM} 2$, Lemma 1], where we have also removed the restriction $\varphi(0)=0$. 
Lemma 4.2. Let $X$ be a complex Banach space and $\varphi$ be an analytic self-map of $\mathbb{D}$. Then

$$
\mathcal{C}\left[S_{\varphi}(\mu)\right]=\mathcal{C}[\mu] \circ \varphi+\int_{\mathbb{T}} \frac{\overline{\xi(0)}}{1-\overline{\xi \varphi(0)}} d \mu(\xi), \quad \mu \in M(X),
$$

where $S_{\varphi}: M(X) \rightarrow M(X)$ is defined by (4.5). If $\varphi(0)=0$, then

$$
C_{\varphi}(\pi f)=\pi\left(\tilde{C}_{\varphi} f\right), \quad f \in h^{1}(X),
$$

where $\pi: h^{1}(X) \rightarrow C T(X)$ is defined by (4.3) and $\tilde{C}_{\varphi}$ is the corresponding composition map $h^{1}(X) \rightarrow h^{1}(X)$.

Proof. Let $0<r<1$ and $z \in \mathbb{D}$. By using the fact that $x^{*}\left(\int_{\mathbb{T}} g d \mu\right)=$ $\int_{\mathbb{T}} g d\left(x^{*} \circ \mu\right)$ whenever $g: \mathbb{T} \rightarrow \mathbb{C}$ is a continuous map, $\mu \in M(X)$ and $x^{*} \in X^{*}$, we get from the standard Fubini theorem that

$$
\begin{aligned}
\int_{\mathbb{T}} \frac{(\mathcal{P}[\mu] \circ \varphi)(r \zeta)}{1-\bar{\zeta} z} d m(\zeta) & =\int_{\mathbb{T}} \frac{1}{1-\bar{\zeta} z} \int_{\mathbb{T}} \frac{1-|\varphi(r \zeta)|^{2}}{|1-\bar{\xi} \varphi(r \zeta)|^{2}} d \mu(\xi) d m(\zeta) \\
& =\int_{\mathbb{T}} \int_{\mathbb{T}} \frac{1-|\varphi(r \zeta)|^{2}}{(1-\bar{\zeta} z)|1-\bar{\xi} \varphi(r \zeta)|^{2}} d m(\zeta) d \mu(\xi) .
\end{aligned}
$$

From Cauchy's theorem

$$
\int_{\mathbb{T}} \frac{1}{(1-\bar{\zeta} z)(1-\bar{\xi} \varphi(r \zeta))} d m(\zeta)=\frac{1}{1-\bar{\xi} \varphi(r z)}
$$

and (since the integrand is anti-analytic in $\zeta$ )

$$
\int_{\mathbb{T}} \frac{\overline{\xi(r \zeta)}}{(1-\bar{\zeta} z)(1-\xi \overline{\varphi(r \zeta)})} d m(\zeta)=\frac{\overline{\xi(0)}}{1-\overline{\xi(0)}}
$$

for $\xi \in \mathbb{T}$. By substituting $\frac{1-|\varphi(r \zeta)|^{2}}{|1-\bar{\xi} \varphi(r \zeta)|^{2}}=\frac{1}{1-\bar{\xi} \varphi(r \zeta)}+\frac{\xi \overline{\varphi(r \zeta)}}{1-\xi \overline{\varphi(r \zeta)}}$ above we get

$$
\begin{aligned}
\int_{\mathbb{T}} \frac{(\mathcal{P}[\mu] \circ \varphi)(r \zeta)}{1-\bar{\zeta} z} d m(\zeta) & =\int_{\mathbb{T}} \frac{1}{1-\bar{\xi} \varphi(r z)} d \mu(\xi)+\int_{\mathbb{T}} \frac{\overline{\xi \overline{\varphi(0)}}}{1-\overline{\xi(0)}} d \mu(\xi) \\
& =\mathcal{C}[\mu](\varphi(r z))+\int_{\mathbb{T}} \frac{\overline{\xi \varphi(0)}}{1-\overline{\xi(0)}} d \mu(\xi),
\end{aligned}
$$

so that

$$
\lim _{r \rightarrow 1} \int_{\mathbb{T}} \frac{(\mathcal{P}[\mu] \circ \varphi)(r \zeta)}{1-\bar{\zeta} z} d m(\zeta)=\mathcal{C}[\mu](\varphi(z))+\int_{\mathbb{T}} \frac{\overline{\xi(0)}}{1-\overline{\xi(0)}} d \mu(\xi) .
$$

We next claim that for each $x^{*} \in X^{*}$ and $z \in \mathbb{D}$ there is a sequence $\left(r_{n}\right)$, depending on $x^{*}$ and $z$, so that $\lim _{n \rightarrow \infty} r_{n}=1$ and

$$
\begin{aligned}
\lim _{n \rightarrow \infty}\left\langle x^{*}, \int_{\mathbb{T}} \frac{(\mathcal{P}[\mu] \circ \varphi)\left(r_{n} \zeta\right)}{1-\bar{\zeta} z} d m(\zeta)\right\rangle & =\left\langle x^{*}, \int_{\mathbb{T}} \frac{1}{1-\bar{\zeta} z} d\left(S_{\varphi}(\mu)\right)(\zeta)\right\rangle \\
& =\left\langle x^{*}, \mathcal{C}\left[S_{\varphi}(\mu)\right](z)\right\rangle .
\end{aligned}
$$

Clearly we get (4.6) by combining (4.8) and (4.9) for $x^{*} \in X^{*}$.

The argument for (4.9) is suggested by the proof of Theorem 2.1 given in [H1, p. 17], and we reproduce the details here for completeness. We consider $X$ as a closed subspace of $X^{* *}$, whence also $M(X) \subset M\left(X^{* *}\right)$. Recall that $M\left(X^{* *}\right)=C\left(\mathbb{T}, X^{*}\right)^{*}$ by Singer's representation theorem [Si] (see also [H4]), 
where the duality is given by $\langle\langle g, \mu\rangle\rangle=\int_{\mathbb{T}}\langle g, d \mu\rangle$ for $g \in C\left(\mathbb{T}, X^{*}\right)$ and $\mu \in M\left(X^{* *}\right)$. Here $C\left(\mathbb{T}, X^{*}\right)$ is the sup-normed space of the continuous functions $\mathbb{T} \rightarrow X^{*}$. Above $\int_{\mathbb{T}}\langle h, d \mu\rangle=\sum_{j=1}^{m}\left\langle\mu\left(E_{j}\right), y_{j}^{*}\right\rangle$ for simple functions $h=\sum_{j=1}^{m} y_{j}^{*} 1_{E_{j}}: \mathbb{T} \rightarrow X^{*}$, and this extends by approximation in the supnorm to $g \in C\left(\mathbb{T}, X^{*}\right)$.

Let $\left(\nu_{r}\right)_{0<r<1}$ be the bounded net in $M\left(X^{* *}\right)$ given by $d \nu_{r}=\left(\mathcal{P}[\mu] \circ \varphi_{r}\right) d m$ for $0<r<1$ and $\mu \in M(X)$. By Alaoglu's theorem $\left(\nu_{r}\right)$ has a $w^{*}$-cluster point $\nu \in M\left(X^{* *}\right)$. Let $P_{z}(\zeta)=\frac{1-|z|^{2}}{|\zeta-z|^{2}}$ denote the Poisson kernel at $z \in \mathbb{D}$. The map $x^{*} P_{z}(\cdot) \in C\left(\mathbb{T}, X^{*}\right)$ for any fixed $x^{*} \in X^{*}$ and $z \in \mathbb{D}$. Hence there is a sequence $\left(r_{n}\right)$ so that $r_{n} \rightarrow 1$ as $n \rightarrow \infty$, and

$$
\left\langle\left\langle x^{*} P_{z}, \nu_{r_{n}}\right\rangle\right\rangle \rightarrow\left\langle\left\langle x^{*} P_{z}, \nu\right\rangle\right\rangle=\int_{\mathbb{T}}\left\langle x^{*} P_{z}(\zeta), d \nu(\zeta)\right\rangle=\left\langle x^{*}, \mathcal{P}[\nu](z)\right\rangle
$$

as $n \rightarrow \infty$. On the other hand,

$$
\begin{aligned}
\left\langle\left\langle x^{*} P_{z}, \nu_{r_{n}}\right\rangle\right\rangle & =\int_{\mathbb{T}}\left\langle x^{*} P_{z}(\zeta), d \nu_{r_{n}}(\zeta)\right\rangle=\left\langle x^{*}, \int_{\mathbb{T}} P_{z}(\zeta) \mathcal{P}[\mu]\left(\varphi\left(r_{n} \zeta\right)\right) d m(\zeta)\right\rangle \\
& =\left\langle x^{*}, \mathcal{P}[\mu]\left(\varphi\left(r_{n} z\right)\right\rangle \rightarrow\left\langle x^{*},(\mathcal{P}[\mu] \circ \varphi)(z)\right)\right\rangle
\end{aligned}
$$

as $n \rightarrow \infty$. Thus $\left\langle x^{*}, \mathcal{P}[\nu](z)\right\rangle=\left\langle x^{*}, \mathcal{P}\left[S_{\varphi}(\mu)\right](z)\right\rangle$ by (4.5) for every $x^{*} \in X^{*}$ and $z \in \mathbb{D}$, so that $\nu=S_{\varphi}(\mu)$ by uniqueness. This implies that for each $g \in C\left(\mathbb{T}, X^{*}\right)$ there is a sequence $\left(r_{n}\right)$ as above (depending on $g$ ) so that

$$
\int_{\mathbb{T}}\left\langle g, d \nu_{r_{n}}\right\rangle \rightarrow \int_{\mathbb{T}}\left\langle g, d\left(S_{\varphi}(\mu)\right)\right\rangle
$$

as $n \rightarrow \infty$. By applying this fact to the continuous maps $\zeta \mapsto \frac{x^{*}}{1-\bar{\zeta} z}$, where $x^{*} \in X^{*}$ and $z \in \mathbb{D}$ are arbitrary, we get that

$$
\begin{aligned}
\left\langle x^{*}, \int_{\mathbb{T}} \frac{(\mathcal{P}[\mu] \circ \varphi)\left(r_{n} \zeta\right)}{1-\bar{\zeta} z} d m(\zeta)\right\rangle & =\left\langle x^{*}, \mathcal{C}\left[\nu_{r_{n}}\right](z)\right\rangle=\int_{\mathbb{T}}\left\langle\frac{x^{*}}{1-\bar{\zeta} z}, d \nu_{r_{n}}(\zeta)\right\rangle \\
& \rightarrow \int_{\mathbb{T}}\left\langle\frac{x^{*}}{1-\bar{\zeta} z}, d\left(S_{\varphi}(\mu)\right)(\zeta)\right\rangle
\end{aligned}
$$

as $n \rightarrow \infty$. This completes the proof of (4.9), and thus of (4.6).

If $\varphi(0)=0$ then the factorization (4.7) is rather immediate by combining (4.6), (4.3) and (4.5).

Proof of Theorem 4.1. Let $f=\mathcal{C}[\mu] \in C T(X)$. We get from (4.6) that

$$
\begin{aligned}
\|\mathcal{C}[\mu] \circ \varphi\|_{C T(X)} & =\left\|\mathcal{C}\left[S_{\varphi}(\mu)\right]-\int_{\mathbb{T}} \frac{\overline{\xi(0)}}{1-\overline{\xi(0)}} d \mu(\xi)\right\|_{C T(X)} \\
& \leq\left\|S_{\varphi}(\mu)\right\|_{C T(X)}+\frac{|\varphi(0)|}{1-|\varphi(0)|}|\mu|(\mathbb{T}) \\
& \leq \frac{1+2|\varphi(0)|}{1-|\varphi(0)|}\|\mu\|_{M(X)},
\end{aligned}
$$

because $\left\|S_{\varphi}(\mu)\right\|_{M(X)} \leq \frac{1+|\varphi(0)|}{1-|\varphi(0)|}\|\mu\|_{M(X)}$ by Proposition 3.1 and (4.5). We obtain the desired estimate

$$
\left\|C_{\varphi}(f)\right\|_{C T(X)} \leq \frac{1+2|\varphi(0)|}{1-|\varphi(0)|}\|f\|_{C T(X)}
$$


by taking the infimum over $\mu \in M(X)$ with $f=\mathcal{C}[\mu]$.

Cima and Matheson [CM2, Thm. 2] showed that the equivalent condition

(6) $C_{\varphi}$ is (weakly) compact on $C T$

can be added to the Omnibus Theorem stated in the Introduction. The vector-valued version of this result follows now easily. Note that if $C_{\varphi}$ is weakly compact on $C T(X)$ then $X$ must be a reflexive space, since $C_{\varphi}$ preserves the constants $z \mapsto x=\mathcal{C}[x d m]$ for $x \in X$.

Theorem 4.3. Let $X$ be a reflexive Banach space and $\varphi$ be an analytic selfmap of $\mathbb{D}$. Then $C_{\varphi}$ is weakly compact $C T(X) \rightarrow C T(X)$ if and only if condition (5) from the Omnibus Theorem holds.

Proof. Suppose that condition (5) holds. We may assume that $\varphi(0)=0$, since by composing with a suitable Möbius transformation $\psi$ we get a linear isomorphism $C_{\psi}$ on both $C T(X)$ and $h^{1}(X)$. If $\varphi(0)=0$ then $C_{\varphi} \circ \pi=\pi \circ \tilde{C}_{\varphi}$ by (4.7), where $\pi$ is defined by (4.3) and the corresponding composition operator $h^{1}(X) \rightarrow h^{1}(X)$ is denoted by $\tilde{C}_{\varphi}$ for extra clarity. Theorem 3.2 implies that $\tilde{C}_{\varphi}$ is a weakly compact operator on $h^{1}(X)$. Since the map $\pi$ satisfies $B_{C T(X)}=\overline{\pi\left(B_{h^{1}(X)}\right)}$ we get that

$$
C_{\varphi}\left(B_{C T(X)}\right) \subset \overline{\pi\left(\tilde{C}_{\varphi}\left(B_{h^{1}(X)}\right)\right.},
$$

where the right-hand set is weakly compact.

Conversely, fix $x \in X$ and $x^{*} \in X^{*}$ so that $x^{*}(x)=1$. As in the proof of Theorem 3.2 one has the factorization

$$
B \circ C_{\varphi} \circ A=\tilde{C}_{\varphi},
$$

where the operators $A: C T \rightarrow C T(X)$ and $B: C T(X) \rightarrow C T$ are defined by $A(\mathcal{C}[\mu])=x \mathcal{C}[\mu]=\mathcal{C}[x \mu]$ and $B(\mathcal{C}[\nu])=x^{*} \circ \mathcal{C}[\mu]=\mathcal{C}\left[x^{*} \circ \nu\right]$ for $\mu \in M$ and $\nu \in M(X)$. Above $\tilde{C}_{\varphi}$ denotes the corresponding composition map $C T \rightarrow C T$. If $C_{\varphi}$ is weakly compact on $C T(X)$, then $\tilde{C}_{\varphi}$ is weakly compact on $C T$ by (4.10). We get from [CM2, Thm. 2] that $\tilde{C}_{\varphi}$ is also (weakly) compact on $h^{1}$, whence condition (5) holds by the Omnibus Theorem.

\section{Composition operators on WeAK SPACES OF VeCTOR-VALUEd HARMONIC FUNCTIONS}

Let $X$ be a complex Banach space. One may introduce another vectorvalued analogue of the harmonic Hardy spaces $h^{p}$ by departing from the fact that a vector-valued map $f: \mathbb{D} \rightarrow X$ is harmonic precisely when it is weakly harmonic. The harmonic function $f: \mathbb{D} \rightarrow X$ belongs to the weak harmonic Hardy space $w h^{p}(X)$ for $1 \leq p<\infty$ if

$$
\|f\|_{w h^{p}(X)}=\sup \left\{\left\|x^{*} \circ f\right\|_{h^{p}}: x^{*} \in X^{*},\left\|x^{*}\right\| \leq 1\right\}<\infty .
$$

These spaces were studied e.g. by Blasco [B1], who characterized the boundary values of the functions $f \in w h^{p}(X)$, as well as in [FGR1], [FGR2].

It follows from Theorem 5.3 below that for any reflexive space $X$ the composition operator $C_{\varphi}$ is weakly compact on $w h^{1}(X)$ precisely when it is compact on $h^{1}$. This provides a counterpart in the harmonic setting of some of the results and techniques of Bonet, Domański and Lindström [BDL], 
who studied composition operators on weak spaces of vector-valued analytic functions (see also $[\mathrm{BF}]$ for a locally convex variant). Here the tools are quite different from section 3, and the spaces $w h^{p}(X)$ and $h^{p}(X)$ also differ for any infinite-dimensional space $X$ (see section 6 ). These techniques actually apply to a large class of weak spaces of vector-valued harmonic functions.

We first recall and describe the setting. Let $\left(E,\|\cdot\|_{E}\right)$ be a Banach space of harmonic functions $\mathbb{D} \rightarrow \mathbb{C}$ so that

(i) $E$ contains the constants,

(ii) $\left(B_{E}, \tau\right)$ is compact, where $B_{E}=\left\{x \in E:\|x\|_{E} \leq 1\right\}$ and $\tau$ is the topology of uniform convergence on compact subsets of $\mathbb{D}$.

The following basic facts, which closely reflect the analytic case, follow from conditions (i) and (ii).

(iii) Let $\delta_{z}$ be the point evaluation map given by $\delta_{z}(f)=f(z)$ for $f \in E$ and $z \in \mathbb{D}$. Then $\delta_{z} \in E^{*}$, and $\delta_{z} \neq 0$, for $z \in \mathbb{D}$.

(iv) Recall that $\tau$ is the locally convex topology on $E$ generated by the seminorms $\sup _{z \in K}|f(z)|$, where $K \subset \mathbb{D}$ runs through the family of compact subsets. Hence the Dixmier-Ng theorem [N, Thm. 1] implies that $E$ is isometrically isomorphic to $V^{*}$, where $V=\left\{u \in E^{*}: u\right.$ is $\tau$-continuous on $\left.B_{E}\right\}$ is a closed subspace of $E^{*}$. The isometry $j: E \rightarrow V^{*}$ is defined by $(j(f))(v)=$ $v(f)$ for $v \in V$ and $f \in E$. The predual $V$ has the following explicit form.

Lemma 5.1. $V=\left[\delta_{z}: z \in \mathbb{D}\right]$, the closed linear span of $\left\{\delta_{z}: z \in \mathbb{D}\right\}$ in $E^{*}$.

Proof. It is obvious that $\left[\delta_{z}: z \in \mathbb{D}\right] \subset V$. Conversely, the linear span of $\left\{\delta_{z}: z \in \mathbb{D}\right\}$ is dense in $V$ by the Hahn-Banach theorem: if $v^{*}=j(f) \in V^{*}$ satisfies $0=(j(f))\left(\delta_{z}\right)=f(z)$ for $z \in \mathbb{D}$, then $v^{*}=0$.

The space

$$
w E(X)=\left\{f: \mathbb{D} \rightarrow X \mid f \text { harmonic, } x^{*} \circ f \in E \text { for every } x^{*} \in X^{*}\right\}
$$

is the weak space of harmonic functions based on $E$, which is equipped with the norm

$$
\|f\|_{w E(X)}=\sup _{\left\|x^{*}\right\|_{X^{*} \leq 1}}\left\|x^{*} \circ f\right\|_{E} .
$$

The closed graph theorem and condition (ii) easily yield that $x^{*} \mapsto x^{*} \circ f$ defines a bounded operator $X^{*} \rightarrow E$ for $f \in w E(X)$, so that $\|f\|_{w E(X)}<\infty$. It is a basic fact that $w E(X)$ is isometric to a space of bounded linear operators (thus $w E(X)$ is always a Banach space). For $w h^{p}(X)$ such an isometry was found directly in [B1, Thm. 9].

Lemma 5.2. Let $X$ be a complex Banach space. Then there is an isometric isomorphism $\chi: L(V, X) \rightarrow w E(X)$ so that

$$
(\chi(T))(z)=T\left(\delta_{z}\right), \quad\left(\chi^{-1}(f)\right)\left(\delta_{z}\right)=f(z)
$$

for $T \in L(V, X), f \in w E(X)$ and $z \in \mathbb{D}$.

Proof. Put $\Delta(z)=\delta_{z}$ for $z \in \mathbb{D}$, so that $\Delta$ maps $\mathbb{D}$ into $V$ by Lemma 5.1. Moreover, $\Delta$ is a (weakly) harmonic map $\mathbb{D} \rightarrow V$, since $z \mapsto(\Delta(z))(f)=$ $f(z)$ is harmonic for every $f \in E=V^{*}$. Clearly

$$
\|\Delta\|_{w E(V)}=\sup _{\left\|v^{*}\right\|_{V^{*} \leq 1}}\left\|v^{*} \circ \Delta\right\|_{E}=\sup _{\|g\|_{E} \leq 1}\|j(g) \circ \Delta\|_{E}=1,
$$


since $j(g) \circ \Delta=g$.

Put $\chi(T)=T \circ \Delta$ for $T \in L(V, X)$. The composite $T \circ \Delta: \mathbb{D} \rightarrow X$ is obviously harmonic, and

$$
\left\|x^{*} \circ T \circ \Delta\right\|_{E} \leq\|T\| \cdot \sup _{\left\|v^{*}\right\|_{V^{*}} \leq 1}\left\|v^{*} \circ \Delta\right\|_{E}=\|T\|
$$

for $x^{*} \in B_{X^{*}}$. Hence $\chi: L(V, X) \rightarrow w E(X)$ and $\|\chi\| \leq 1$. Moreover, $(\chi(T))(z)=(T \circ \Delta)(z)=T\left(\delta_{z}\right)$ for $T \in L(V, X)$ and $z \in \mathbb{D}$.

Define $\psi: w E(X) \rightarrow L\left(V, X^{* *}\right)$ by $(\psi(g)(v))\left(x^{*}\right)=\left(j\left(x^{*} \circ g\right)\right)(v)$ for $g \in w E(X), v \in V$ and $x^{*} \in X^{*}$. Thus $\psi$ is a bounded linear map, and $\|\psi\| \leq 1$, since

$$
\left|(\psi(g)(v))\left(x^{*}\right)\right| \leq\|j\|\left\|x^{*} \circ g\right\|_{E}\|v\|_{V} \leq\left\|x^{*}\right\|_{X^{*}}\|g\|_{w E(X)}\|v\|_{V} .
$$

Moreover, $\left(\psi(g)\left(\delta_{z}\right)\right)\left(x^{*}\right)=\left(j\left(x^{*} \circ g\right)\right)\left(\delta_{z}\right)=x^{*}(g(z))$ for $x^{*} \in X^{*}$, so that $(\psi(g))\left(\delta_{z}\right)=g(z) \in X$ (here $X$ is considered as the canonical subspace of $\left.X^{* *}\right)$ for $g \in w E(X)$ and $z \in \mathbb{D}$. Since $V=\left[\delta_{z}: z \in \mathbb{D}\right]$ by Lemma 5.1, we get that $\psi(g)(V) \subset X$ and we may view $\psi$ as a map $w E(X) \rightarrow L(V, X)$.

It remains to check that $\psi \circ \chi=I_{L(V, X)}$ and $\chi \circ \psi=I_{w E(X)}$, respectively. For $T \in L(V, X)$ and $\delta_{z} \in V$ one gets from $(5.1)$ that $((\psi \circ \chi)(T))\left(\delta_{z}\right)=$ $(\chi(T))(z)=T\left(\delta_{z}\right)$. Hence $(\psi \circ \chi)(T)=T$ by Lemma 5.1. Moreover, for $f \in w E(X)$ and $z \in \mathbb{D}$ one obtains from (5.1) that $((\chi \circ \psi)(f))(z)=$ $\left(\chi(\psi(f))(z)=(\psi(f))\left(\delta_{z}\right)=f(z)\right.$, so that $(\chi \circ \psi)(f)=f$.

We collect our results about composition operators on $w E(X)$ into the following theorem, where part (3) is the main result of this section.

Theorem 5.3. Let $X$ be a complex Banach space and $\varphi$ be an analytic selfmap of $\mathbb{D}$.

(1) If $C_{\varphi}$ is bounded $E \rightarrow E$, then $C_{\varphi}$ is also bounded $w E(X) \rightarrow w E(X)$.

(2) If $C_{\varphi}$ is weakly compact $w E(X) \rightarrow w E(X)$, then $X$ is reflexive and $C_{\varphi}$ is weakly compact $E \rightarrow E$.

(3) Let $X$ be reflexive and suppose that $C_{\varphi}$ is a compact operator $E \rightarrow E$. Then $C_{\varphi}$ is weakly compact $w E(X) \rightarrow w E(X)$.

Proof. For clarity we again denote the composition operator on $E$ by $\tilde{C}_{\varphi}$.

(1) If $f \in w E(X)$ and $x^{*} \in X^{*}$, then $x^{*} \circ f \in E$ and

$$
\left\|x^{*} \circ\left(C_{\varphi} f\right)\right\|_{E}=\left\|\tilde{C}_{\varphi}\left(x^{*} \circ f\right)\right\|_{E} \leq\left\|\tilde{C}_{\varphi}\right\| \cdot\left\|x^{*} \circ f\right\|_{E},
$$

so that $\left\|C_{\varphi} f\right\|_{w E(X)} \leq\left\|\tilde{C}_{\varphi}\right\| \cdot\|f\|_{w E(X)}$. Hence $C_{\varphi}$ is bounded $w E(X) \rightarrow$ $w E(X)\left(\right.$ and $\left.\left\|C_{\varphi}\right\| \leq\left\|\tilde{C}_{\varphi}\right\|\right)$.

(2) Assume that $C_{\varphi}$ is weakly compact $w E(X) \rightarrow w E(X)$. Similarly as in (3.2) and (4.10) there is a factorization $B \circ C_{\varphi} \circ A=\tilde{C}_{\varphi}$, whence $\tilde{C_{\varphi}}$ is weakly compact $E \rightarrow E$. Let $S: X \rightarrow w E(X)$ be the linear map $x \mapsto f_{x}$, where $f_{x}(z) \equiv x$ for $x \in X$, and let $T: w E(X) \rightarrow X$ be the evaluation operator $f \mapsto f(0)$. Note that $T$ is bounded, since $\left|x^{*}(f(0))\right| \leq\left\|\delta_{0}\right\| \cdot\left\|x^{*} \circ f\right\|_{E}$ by (iii) for $f \in w E(X)$ and $x^{*} \in B_{X^{*}}$, so that $\|f(0)\|_{X} \leq\left\|\delta_{0}\right\| \cdot\|f\|_{w E(X)}$. Obviously $I_{X}=T \circ C_{\varphi} \circ S$, whence $I_{X}$ is weakly compact (and $X$ is reflexive).

(3) The adjoint $\left(\tilde{C}_{\varphi}\right)^{*}: E^{*} \rightarrow E^{*}$ satisfies $\left(\tilde{C}_{\varphi}\right)^{*}\left(\delta_{z}\right)=\delta_{\varphi(z)}$ for $z \in \mathbb{D}$, since $\left\langle f,\left(\tilde{C}_{\varphi}\right)^{*}\left(\delta_{z}\right)\right\rangle=\left\langle f \circ \varphi, \delta_{z}\right\rangle=\left\langle f, \delta_{\varphi(z)}\right\rangle$ for $f \in w E(X)$. Hence 
$\left(\tilde{C}_{\varphi}\right)^{*} V \subset V$ by Lemma 5.1. We claim that

$$
C_{\varphi}=\chi \circ W_{\varphi} \circ \psi
$$

where $W_{\varphi}$ is the operator composition map

$$
T \mapsto i d_{X} \circ T \circ\left(\tilde{C}_{\varphi}\right)^{*}{ }_{\mid V} ; \quad L(V, X) \rightarrow L(V, X),
$$

and $\chi: L(V, X) \rightarrow w E(X), \psi=\chi^{-1}: w E(X) \rightarrow L(V, X)$ are the isometries given by Lemma 5.2. Indeed, by (5.1)

$$
\begin{aligned}
\left(\left(\chi \circ W_{\varphi} \circ \psi\right)(f)\right)(z) & =\chi\left(\psi(f) \circ\left(\tilde{C}_{\varphi}\right)^{*}{ }_{\mid V}\right)(z)=\psi(f)\left(\left(\tilde{C}_{\varphi}\right)^{*}{ }_{\mid V}\right)\left(\delta_{z}\right) \\
& =\psi(f)\left(\delta_{\varphi(z)}\right)=f(\varphi(z))
\end{aligned}
$$

for $f \in w E(X)$ and $z \in \mathbb{D}$.

By our assumptions $I_{X}$ is weakly compact and $\left(\tilde{C}_{\varphi}\right)^{*}$ is compact. It follows from [ST, Thm. 2.9] that the map $W_{\varphi}$ in (5.3) is weakly compact on $L(V, X)$. Hence $C_{\varphi}: w E(X) \rightarrow w E(X)$ is weakly compact by (5.2).

The linearization trick (5.2) for composition operators on $w E(X)$ is due to Bonet, Domański and Lindström [BDL]. The analytic version [BDL, Prop. 11] of Theorem 5.3 applies to a large number of weak spaces of analytic functions, including the weak Hardy spaces $w H^{p}(X)$ for $1 \leq p<\infty$, the weak Bergman spaces $w B_{p}(X)$ for $1 \leq p<\infty$, the weighted weak $H^{\infty}$ spaces $w B_{\infty}^{v}(X)$ (see [BDL, Cor. 15]), the weak Bloch space $w \mathcal{B}(X)$ (see [BDL, Cor. 12]), and $w B M O A(X)$ (see also [L, Section 5]). In [BDL, Section 5] the authors only discussed $w B_{\infty}^{v}(X)$ and $w \mathcal{B}(X)$ explicitly. In these cases $w E(X)=E(X)$, where the strong spaces $E(X)$ are defined in a natural manner. Such an equality is quite exceptional (see section 6 ).

The following examples were suggested by sections 3 and 4 . Recall that $h^{\infty}$ is the Banach space of the bounded harmonic functions $f: \mathbb{D} \rightarrow \mathbb{C}$ equipped with the supremum norm.

Example 5.4. $h^{p}(1 \leq p \leq \infty)$ and $C T$ satisfy conditions (i) and (ii).

Proof. We recall the idea for $C T$ (cf. also [CMR, Section 5]). Suppose that $\left(f_{n}\right) \subset B_{C T}$ and pick a bounded sequence $\left(\mu_{n}\right) \subset M$, so that $f_{n}=\mathcal{C}\left[\mu_{n}\right]$ for $n \in \mathbb{N}$. By $w^{*}$-sequential compactness there is a subsequence $\left(\mu_{n_{k}}\right)$ so that $\mu_{n_{k}} \stackrel{w^{*}}{\longrightarrow} \mu$ in $M$ as $k \rightarrow \infty$. Since the Cauchy kernel $\zeta \mapsto \frac{1}{1-\bar{\zeta} z}$ is continuous we get that $f_{n_{k}}(z)=\mathcal{C}\left[\mu_{n_{k}}\right](z) \rightarrow \mathcal{C}[\mu](z)$ as $k \rightarrow \infty$ for $z \in \mathbb{D}$. This implies that $f_{n_{k}} \stackrel{\tau}{\longrightarrow} \mathcal{C}[\mu]$. The other cases are easier, since $h^{1}=\mathcal{P} M$ and $h^{p}=\mathcal{P} L^{p}$ for $1<p \leq \infty$.

Strictly speaking, composition operators on $w C T(X)$ are covered already by the analytic case [BDL, Section 5]. Note that if $E$ is a Banach space consisting of analytic functions $\mathbb{D} \rightarrow \mathbb{C}$ which satisfies conditions (i) and (ii), then the map $\Delta: \mathbb{D} \rightarrow V$ from Lemma 5.2 is (weakly) analytic. This fact provides the connection between Theorem 5.3 and [BDL, Section 5].

Among other interesting weak spaces $w E(X)$ of harmonic functions one finds those obtained by taking $E$ as a harmonic Bergman space (see [C, 18.1]) or the harmonic BMO-space $\mathcal{P}(B M O)$. 
Remarks. (i) Theorem 5.3 is sharp in the following sense: there are spaces $E$ satisfying conditions (i) and (ii), and weakly compact maps $C_{\varphi}: E \rightarrow E$, for which $C_{\varphi}$ fails to be weakly compact $w E(X) \rightarrow w E(X)$. It is enough to observe that the weak space $w h^{2}\left(\ell^{2}\right)$ is non-reflexive. In fact, $w h^{2}\left(\ell^{2}\right)=$ $L\left(V, \ell^{2}\right)$ by Lemma 5.2 , where $V=\left[\delta_{z}: z \in \mathbb{D}\right] \subset\left(h^{2}\right)^{*}$ is isometric to $\ell^{2}$. Hence, if $\varphi(z)=z$ for $z \in \mathbb{D}$, then $C_{\varphi}=I_{w h^{2}\left(\ell^{2}\right)}$ is not weakly compact. Note that by contrast $h^{2}\left(\ell^{2}\right)$ is a Hilbert space.

(ii) If $C_{\varphi}$ is compact on $E$ and $X$ does not contain any isomorphic copies of $l^{1}$, then $C_{\varphi}$ is weakly conditionally compact $w E(X) \rightarrow w E(X)$. For this fact one follows the argument in Theorem 5.3, but one applies [LS, Cor. 2.13] instead of [ST, Thm. 2.9].

Clearly $w h^{\infty}(X)=h^{\infty}(X)$, with equal norms, for any Banach space $X$. This fact leads in tandem with Theorem 5.3 to a straightforward characterization of the weakly compact maps $C_{\varphi}$ on $h^{\infty}(X)$, which we include for completeness. (Alternatively, for the converse implication below one may also argue directly as in Lemma 3.3.)

Proposition 5.5. Let $X$ be a reflexive space, and $\varphi$ an analytic self-map of $\mathbb{D}$. Then $C_{\varphi}$ is weakly compact $h^{\infty}(X) \rightarrow h^{\infty}(X)$ if and only if $\|\varphi\|_{\infty}<1$.

Proof. If $C_{\varphi}$ is weakly compact $h^{\infty}(X) \rightarrow h^{\infty}(X)$, then it is also weakly compact on $h^{\infty}$ by the familiar argument. It follows that $C_{\varphi}$ is actually compact on $h^{\infty}$. Indeed, $C_{\varphi}$ is then weakly compact on the invariant subspace $H^{\infty}$, and hence compact on $H^{\infty}$ (see the proof of [LST, Thm. 6]). This implies that $\|\varphi\|_{\infty}<1$, see [CoM, Ex. 3.2.2]. Conversely, $C_{\varphi}$ is compact on $h^{\infty}$ if $\|\varphi\|_{\infty}<1$ (apply the argument of Lemma 3.3 for $X=\mathbb{C}$ ). Theorem 5.3.(3) and the equality $w h^{\infty}(X)=h^{\infty}(X)$ imply that $C_{\varphi}$ is weakly compact $h^{\infty}(X) \rightarrow h^{\infty}(X)$.

\section{Strong Vs. WEAK SPACES OF VECTOR-VALUEd HARMONiC FUNCTIONS}

In this section we exhibit concrete functions which demonstrate how $w h^{p}(X)$ and $h^{p}(X)$ differ for any complex infinite-dimensional Banach space $X$ and $1 \leq p<\infty$, and how $w C T(X)$ and $C T(X)$ differ e.g. if $X$ is a $\mathrm{K}$-convex space. Such examples are interesting in themselves, but our principal aim is to emphasize that the setting of Theorem 5.3 is quite different from those of sections 3 and 4 .

Clearly $\|f\|_{w h^{p}(X)} \leq\|f\|_{h^{p}(X)}$ for $f \in h^{p}(X)$, so that $h^{p}(X)$ is continuously embedded in $w h^{p}(X)$. It will turn out that the norms $\|\cdot\|_{w h^{p}(X)}$ and $\|\cdot\|_{h^{p}(X)}$ are never equivalent on $h^{p}(X)$ for $1 \leq p<\infty$. Blasco [B1, Cor. 23] observed that $h^{1}(C(\mathbb{T})) \varsubsetneqq w h^{1}(C(\mathbb{T}))$ and $h^{p}\left(L^{p^{\prime}}\right) \varsubsetneqq w h^{p}\left(L^{p^{\prime}}\right)$ for $1<p<$ $\infty$, where $p^{\prime}=\frac{p}{p-1}$. Subsequently Freniche, Garcia-Vazguez and RodriguezPiazza exhibited functions $f \in w h^{p}(X) \backslash h^{p}(X)$ and $g \in w H^{p}(X) \backslash H^{p}(X)$ for $1 \leq p<\infty$ and any $X$, see [FGR1, Prop. 4] and [FGR2, Cor. 9 and Cor. 12]. The first author computed [L, Example 5.1] by different means that

$$
\left\|f_{n}\right\|_{w B M O A(X)} \leq 1,\left\|f_{n}\right\|_{B M O A(X)} \geq C(\log n)^{1 / 2}, \quad n \in \mathbb{N},
$$


where $f_{n}(z)=\sum_{k=1}^{n} \frac{z^{k}}{\sqrt{k}} x_{k}^{(n)}(z \in \mathbb{D})$ are concrete analytic polynomials, and $x_{1}^{(n)}, \ldots, x_{n}^{(n)} \in X$ are chosen as in Example 6.1 below. These estimates extend to the $H^{p}$-norms by appealing to the John-Nirenberg theorem (see [L, Example 5.1]).

The examples in [FGR1] and [FGR2] depend on several non-trivial facts, but we will here see the difference between $h^{p}(X)$ and $w h^{p}(X)$ (respectively, $H^{p}(X)$ and $\left.w H^{p}(X)\right)$ using fairly minimal tools. We first compute these norms for certain vector-valued lacunary polynomials. Similar ideas will also be useful for the $C T$-norms.

Example 6.1. Let $X$ be a complex infinite-dimensional Banach space, $1 \leq$ $p<\infty$, and $n \in \mathbb{N}$. Fix a linear isomorphism $T_{n}: \ell_{2}^{n} \rightarrow\left[x_{1}^{(n)}, \ldots, x_{n}^{(n)}\right]$, where $\left[x_{1}^{(n)}, \ldots, x_{n}^{(n)}\right] \subset X$, so that $\left\|T_{n}\right\|=1$ and $\left\|T_{n}^{-1}\right\| \leq 2$ (this is possible by Dvoretzky's theorem, see e.g. [DJT, Thm. 19.1]). Here $x_{k}^{(n)}=T_{n} e_{k}$ for $k=1, \ldots, n$, where $\left(e_{1}, \ldots, e_{n}\right)$ is an orthonormal basis of $\ell_{2}^{n}$. Put $f_{n}(z)=$ $\sum_{k=1}^{n} x_{k}^{(n)} z^{2^{k}}$ for $z \in \mathbb{D}$. Then

$$
\begin{gathered}
a_{p} \leq\left\|f_{n}\right\|_{w H^{p}(X)} \leq b_{p}, \\
\left\|f_{n}\right\|_{H^{p}(X)} \geq \frac{1}{2} n^{1 / 2},
\end{gathered}
$$

where the constants $a_{p}, b_{p}>0$ are independent of $X$ and $n$.

Proof. Our starting point is the following fact about lacunary series (see [Z, Thm. I.V.8.20]): For $0<p<\infty$ there are constants $a_{p}, b_{p}>0$ so that

$$
a_{p}\left(\sum_{k=1}^{n}\left|c_{k}\right|^{2}\right)^{1 / 2} \leq\left\|\sum_{k=1}^{n} c_{k} z^{2^{k}}\right\|_{H^{p}} \leq b_{p}\left(\sum_{k=1}^{n}\left|c_{k}\right|^{2}\right)^{1 / 2}
$$

for all scalars $c_{1}, \ldots, c_{n}$ and all $n \in \mathbb{N}$.

If $x^{*} \in B_{X^{*}}$, then by (6.3) one has that

$$
\begin{aligned}
\left\|x^{*} \circ f_{n}\right\|_{H^{p}} & =\left\|\sum_{k=1}^{n} x^{*}\left(x_{k}^{(n)}\right) z^{2^{k}}\right\|_{H^{p}} \leq b_{p}\left(\sum_{k=1}^{n}\left|x^{*}\left(x_{k}^{(n)}\right)\right|^{2}\right)^{1 / 2} \\
& =b_{p}\left(\sum_{k=1}^{n}\left|T_{n}^{*} y^{*}\left(e_{k}\right)\right|^{2}\right)^{1 / 2}=b_{p}\left\|T_{n}^{*} y^{*}\right\| \leq b_{p},
\end{aligned}
$$

where $y^{*}$ is $x^{*}$ restricted to $\left[x_{1}^{(n)}, \ldots, x_{n}^{(n)}\right]$. Thus $\left\|f_{n}\right\|_{w h^{p}(X)} \leq b_{p}$. For the converse estimate pick a norm- 1 functional $y^{*} \in\left[x_{1}^{(n)}, \ldots, x_{n}^{(n)}\right]^{*}$ so that $\left\|T_{n}^{*} y^{*}\right\|=\left\|T_{n}\right\|$. One gets as above from (6.3) that

$$
a_{p}\left\|T_{n}\right\|=a_{p}\left(\sum_{k=1}^{n}\left|y^{*}\left(x_{k}^{(n)}\right)\right|^{2}\right)^{1 / 2} \leq\left\|x^{*} \circ f_{n}\right\|_{H^{p}} \leq\left\|f_{n}\right\|_{w H^{p}(X)},
$$

where $x^{*} \in X^{*}$ is a norm-1 extension of $y^{*}$.

Let $0<r<1$. Since the linear isomorphism $T_{n}: \ell_{2}^{n} \rightarrow\left[x_{1}^{(n)}, \ldots, x_{n}^{(n)}\right]$ satisfies $\left\|T_{n}^{-1}\right\| \leq 2$ one obtains that

$$
\left\|\left(f_{n}\right)_{r}\right\|_{L^{p}(X)}^{p}=\int_{\mathbb{T}}\left\|\sum_{k=1}^{n} x_{k}^{(n)} r^{2^{k}} \zeta^{2^{k}}\right\|_{X}^{p} d m(\zeta) \geq \frac{1}{2^{p}}\left(\sum_{k=1}^{n} r^{2 \cdot 2^{k}}\right)^{p / 2},
$$


whence $\left\|f_{n}\right\|_{H^{p}(X)} \geq \frac{1}{2} n^{1 / 2}$ by letting $r \rightarrow 1$.

Remarks. (i) Note that lacunarity is not used for the estimate (6.2). If $1 \leq p \leq 2$ then lacunarity is not needed for the upper estimate in (6.1), since $\left\|\sum_{k=1}^{n} c_{k} z^{k}\right\|_{H^{p}} \leq\left(\sum_{k=1}^{n}\left|c_{k}\right|^{2}\right)^{1 / 2}$ for any $c_{1}, \ldots, c_{n}$ by Hölder's inequality.

(ii) The lacunary polynomials from Example 6.1 can also be used to recover [L, Example 5.1] by following that argument.

We next use Example 6.1 to exhibit a concrete analytic function $f: \mathbb{D} \rightarrow X$ for any given $X$ and $p \in[1, \infty)$, so that $f \in w h^{p}(X) \backslash h^{p}(X)$. The trick of blocking a sequence of euclidean copies is familiar from e.g. [DG, Example 4] and [FGR1], [FGR2, Thm. 11] in somewhat similar contexts.

Example 6.2. Let $X$ be any complex infinite-dimensional Banach space, and $1 \leq p<\infty$. Then there is an analytic map $f: \mathbb{D} \rightarrow X$ for which $f \in w H^{p}(X) \backslash H^{p}(X)$ (and consequently also $f \in w h^{p}(X) \backslash h^{p}(X)$ ).

Proof. Fix a normalized basic sequence $\left(v_{n}\right)$ in $X$, see [LT, 1.a.5]. Pick an increasing sequence $\left(m_{n}\right) \subset \mathbb{N}$, so that $m_{n+1}-m_{n}$ is big enough to ensure that $F_{n}=\left[v_{m_{n}+1}, \ldots, v_{m_{n+1}}\right]$ contains a 2-isomorphic copy of $\ell_{2}^{2^{n}}$ for each $n \in \mathbb{N}$. This is again possible by Dvoretzky's theorem. Let $E_{n}=$ $\left[y_{1}^{(n)}, \ldots, y_{2^{n}}^{(n)}\right] \subset F_{n}$ be the resulting $\ell_{2}^{2^{n}}$-copy, normalized as in Example 6.1, and put

$$
f_{n}(z)=\sum_{k=1}^{2^{n}} y_{k}^{(n)} z^{2^{k}}, \quad z \in \mathbb{D}
$$

for $n \in \mathbb{N}$. Thus

$$
\left\|f_{n}\right\|_{w H^{p}(X)} \leq b_{p}, \quad\left\|f_{n}\right\|_{H^{p}(X)} \geq 2^{n / 2-1},
$$

by (6.1) and (6.2), where $b_{p}>0$ is independent of $n \in \mathbb{N}$.

Fix $\alpha \in\left(0, \frac{1}{2}\right)$, and consider the map $f: \mathbb{D} \rightarrow X$ defined by $f(z)=$ $\sum_{n=1}^{\infty} 2^{-\alpha n} f_{n}(z)$ for $z \in \mathbb{D}$. Recall that $\|g(z)\|_{X} \leq \frac{1}{1-|z|}\|g\|_{w H^{p}(X)}$ holds for any maps $g \in w H^{p}(X), z \in \mathbb{D}$ and $1 \leq p<\infty$, cf. [CoM, p. 11]. Since $\sum_{n=1}^{\infty} 2^{-\alpha n}\left\|f_{n}\right\|_{w H^{p}(X)}<\infty$ we get that $\sum_{n=1}^{m} 2^{-\alpha n} f_{n} \rightarrow f$ uniformly on the compact subsets of $\mathbb{D}$ as $m \rightarrow \infty$. Hence $f$ is analytic and $f \in w H^{p}(X)$. Note further that $\|f\|_{w H^{p}(X)}=\|f\|_{w h^{p}(X)}$.

Consider the closed linear span $M=\left[\cup_{n=1}^{\infty} E_{n}\right] \subset X$, and observe that $\left(E_{n}\right)$ is a finite-dimensional Schauder decomposition for $M$, see [LT, 1.g]. Thus there is a constant $c>0$ so that

$$
\|x\| \geq c \sup _{n \in \mathbb{N}}\left\|x_{n}\right\|
$$

holds for every $x=\sum_{n=1}^{\infty} x_{n} \in M$, where $x_{n} \in E_{n}$ for $n \in \mathbb{N}$. Note next that $\left\|\sum_{n=1}^{m} 2^{-\alpha n} f_{n}\right\|_{H^{p}(X)}=\left\|\sum_{n=1}^{m} 2^{-\alpha n} f_{n}\right\|_{H^{p}(M)}$ for $m \in \mathbb{N}$, since $\sum_{n=1}^{m} 2^{-\alpha n} f_{n}(z) \in M$ for $z \in \mathbb{D}$. Hence we get from (6.4) that

$$
\begin{aligned}
\left\|\sum_{n=1}^{m} 2^{-\alpha n} f_{n}\right\|_{H^{p}(X)}^{p} & =\lim _{r \rightarrow 1} \int_{\mathbb{T}}\left\|\sum_{n=1}^{m} 2^{-\alpha n} f_{n}(r \zeta)\right\|_{M}^{p} d m(\zeta) \\
& \geq c^{p} 2^{-\alpha m p} \lim _{r \rightarrow 1} \int_{\mathbb{T}}\left\|f_{m}(r \zeta)\right\|_{M}^{p} d m(\zeta) \\
& \geq c^{p} 2^{-p} 2^{p m\left(\frac{1}{2}-\alpha\right)}
\end{aligned}
$$


for $m \in \mathbb{N}$. Since $0<\alpha<\frac{1}{2}$ we deduce that $\left\|\sum_{n=1}^{m} 2^{-\alpha n} f_{n}\right\|_{H^{p}(M)} \rightarrow \infty$ as $m \rightarrow \infty$. This means that $f \notin H^{p}(X)$ (and $f \notin h^{p}(X)$ as well).

We next discuss how the quotient norms $\|\cdot\|_{C T(X)}$ and $\|\cdot\|_{w C T(X)}$ differ, where it is harder to estimate $\|\cdot\|_{C T(X)}$ from below. We will use the formula

$$
\|\mathcal{C}[\mu]\|_{C T\left(X^{*}\right)}=\sup \left\{\left|\int_{\mathbb{T}}\langle g, \mu\rangle\right|: g \in \overline{A_{0}}(X),\|g\|_{C(\mathbb{T}, X)} \leq 1\right\},
$$

which holds for any $\mu \in M\left(X^{*}\right)$ and any Banach space $X$. Here $\overline{A_{0}}(X)=$ $\left\{g \in C(\mathbb{T}, X): \widehat{g}_{n}=0\right.$ for $\left.n>0\right\}$. The identity (6.5) follows from Singer's representation theorem and Theorem 2.1. In fact, the annihilator

$$
\overline{A_{0}}(X)^{\perp}=\overline{M_{a, 0}}\left(X^{*}\right) \equiv\left\{\mu \in M\left(X^{*}\right): \widehat{\mu}_{n}=0 \text { for } n \leq 0\right\},
$$

see e.g. $\left[\mathrm{H} 1\right.$, p. 56]. Thus $\overline{A_{0}}(X)^{*}=C(\mathbb{T}, X)^{*} / \overline{A_{0}}(X)^{\perp}=M\left(X^{*}\right) / \overline{M_{a, 0}}\left(X^{*}\right)$ isometrically in terms of the duality pairing $\langle\langle g, \mu\rangle\rangle=\int_{\mathbb{T}}\langle g, \mu\rangle$ from Singer's theorem. By applying the isometric isomorphism $\mathcal{P}$ we get the isometric identification $C T\left(X^{*}\right)=M\left(X^{*}\right) / \overline{M_{a, 0}}\left(X^{*}\right)=\overline{A_{0}}(X)^{*}$, where the pairing is given by $\langle g, \mathcal{C}[\mu]\rangle=\int_{\mathbb{T}}\langle g, \mu\rangle$ for $\mu \in M\left(X^{*}\right)$ and $g \in \overline{A_{0}}(X)^{*}$. Thus (6.5) is essentially the well-known duality formula.

As an application of (6.5) we compute the asymptotic behaviour of the $w C T$ - and the $C T$-norms for certain $\ell_{p}^{n}$-valued polynomials.

Example 6.3. Let $1 \leq p<\infty$ and $n \in \mathbb{N}$. Put $f_{n}(z)=\sum_{k=1}^{n} e_{k} z^{k}$ for $z \in \mathbb{D}$, where $\left(e_{1}, \ldots, e_{n}\right)$ is the unit vector basis in $\ell_{p}^{n}$. Then

$$
\begin{gathered}
\left\|f_{n}\right\|_{w C T\left(\ell_{p}^{n}\right)} \leq 1, \quad 2 \leq p<\infty, \\
\left\|f_{n}\right\|_{w C T\left(\ell_{p}^{n}\right)} \leq n^{1 / s}, \quad 1 \leq p<2,
\end{gathered}
$$

where $\frac{1}{s}=\frac{1}{p}-\frac{1}{2}$, and

$$
\left\|f_{n}\right\|_{C T\left(\ell_{p}^{n}\right)} \geq n^{1 / p}
$$

Proof. Suppose that $x^{*}=\sum_{k=1}^{n} x^{*}\left(e_{k}\right) e_{k} \in \ell_{p^{\prime}}^{n}$, where $p^{\prime}=\frac{p}{p-1}$ and $\left(e_{1}, \ldots, e_{n}\right)$ also denotes the unit vector basis in $\ell_{p^{\prime}}^{n}$. By arguing as in Example 6.1, and taking into account remark (i) following that example, we get that

$$
\left\|x^{*} \circ f_{n}\right\|_{h^{1}}=\left\|\sum_{k=1}^{n} x^{*}\left(e_{k}\right) z^{k}\right\|_{H^{1}} \leq\left(\sum_{k=1}^{n}\left|x^{*}\left(e_{k}\right)\right|^{2}\right)^{1 / 2} .
$$

If $2 \leq p<\infty$, then $\left(\sum_{k=1}^{n}\left|x^{*}\left(e_{k}\right)\right|^{2}\right)^{1 / 2} \leq\left(\sum_{k=1}^{n}\left|x^{*}\left(e_{k}\right)\right|^{p^{\prime}}\right)^{1 / p^{\prime}}=\left\|x^{*}\right\|$, so that $\left\|f_{n}\right\|_{w C T\left(\ell_{p}^{n}\right)} \leq\left\|f_{n}\right\|_{w h^{1}\left(\ell_{p}^{n}\right)} \leq 1$. If $1<p<2$, then $\left\|x^{*} \circ f_{n}\right\|_{h^{1}} \leq$ $n^{1 / s}\left\|x^{*}\right\|$ by Hölder's inequality, where $\frac{1}{2}=\frac{1}{p^{\prime}}+\frac{1}{s}$ (and hence $\frac{1}{s}=\frac{1}{p}-\frac{1}{2}$ ). This yields (6.7). The case $p=1$ is similar (here $s=\frac{1}{2}$ ).

Consider the map $g: \mathbb{T} \rightarrow \ell_{p^{\prime}}^{n}$ defined by $g(\zeta)=\sum_{k=1}^{n} \bar{\zeta}^{k} e_{k}$, for which

$$
\|g\|_{C\left(\mathbb{T}, \ell_{p^{\prime}}^{n}\right)}=\sup _{|\zeta|=1}\left\|\sum_{k=1}^{n} \bar{\zeta}^{k} e_{k}\right\|_{\ell_{p^{\prime}}^{n}}=n^{1 / p^{\prime}} .
$$


Thus $n^{-1 / p^{\prime}} g \in B_{\overline{A_{0}}\left(\ell_{p^{\prime}}^{n}\right)}$. Note that $f_{n}=\mathcal{P}\left[\mu_{n}\right]$, where $\mu_{n} \in M\left(\ell_{p}^{n}\right)$ is the absolutely continuous measure $d \mu_{n}=\left(\sum_{s=1}^{n} \zeta^{s} e_{s}\right) d m$ on $\mathbb{T}$. Hence we get from (6.5) that

$$
\begin{aligned}
\left\|f_{n}\right\|_{C T\left(\ell_{p}^{n}\right)} & \geq\left\langle\left\langle n^{-1 / p^{\prime}} g, \mu_{n}\right\rangle\right\rangle \\
& =n^{-1 / p^{\prime}} \int_{\mathbb{T}} \sum_{k, s=1}^{n}\left\langle e_{k}, e_{s}\right\rangle \zeta^{s} \bar{\zeta}^{k} d m(\zeta)=n^{1 / p} .
\end{aligned}
$$

We refer to [DJT, Chapter 13] for the definition and a discussion of Kconvex Banach spaces. We only recall here that $L^{p}$ (and its subspaces) is $\mathrm{K}$-convex for $1<p<\infty$, while $L^{1}$ and $L^{\infty}$ are not $\mathrm{K}$-convex. We will need the following result due to Figiel and Tomczak-Jaegermann: if $X$ is a K-convex space, then there is a constant $C<\infty$ so that for each $n \in \mathbb{N}$ there is a subspace $M_{n} \subset X$ so that $M_{n}$ is 2-isomorphic to $\ell_{2}^{n}$ and $M_{n}$ is $C$-complemented in $X$ (see e.g. [DJT, Thm. 19.3]).

Example 6.4. Suppose that $X$ is an infinite-dimensional complex Banach space, for which there is $p \in[1, \infty)$ and a constant $C<\infty$ so that $X$ contains $C$-isomorphic copies of $\ell_{p}^{n}$ which are $C$-complemented in $X$ for all $n \in \mathbb{N}$. Then there are polynomials $g_{n} \in C T(X)$ such that

$$
\left\|g_{n}\right\|_{w C T(X)} \leq 1, n \in \mathbb{N}, \quad\left\|g_{n}\right\|_{C T(X)} \rightarrow \infty \text { as } n \rightarrow \infty .
$$

The above holds if $X$ is $K$-convex.

Proof. Let $M_{n} \subset X$ be the subspaces guaranteed by the assumptions. Fix linear isomorphisms $S_{n}: \ell_{p}^{n} \rightarrow M_{n}$ and projections $P_{n}: X \rightarrow M_{n}$ so that $\left\|S_{n}\right\|=1,\left\|S_{n}^{-1}\right\| \leq C$ and $\left\|P_{n}\right\| \leq C$ for $n \in \mathbb{N}$.

Let $2 \leq p<\infty$. Consider $g_{n}(z)=S_{n}\left(f_{n}(z)\right)$ for $n \in \mathbb{N}$, where $f_{n}: \mathbb{D} \rightarrow \ell_{p}^{n}$ is the polynomial from Example 6.3. Let $x^{*} \in B_{M_{n}^{*}}$. Then

$$
\left\|x^{*} \circ g_{n}\right\|_{C T}=\left\|\left(x^{*} \circ S_{n}\right) \circ f_{n}\right\|_{C T} \leq\left\|f_{n}\right\|_{w C T\left(\ell_{p}^{n}\right)} \leq 1
$$

by (6.6), so that $\left\|g_{n}\right\|_{w C T(X)} \leq\left\|g_{n}\right\|_{w C T\left(M_{n}\right)} \leq 1$. Recall next that

$$
\left\|g_{n}\right\|_{C T(X)}=\inf \left\{\left\|g_{n}-h\right\|_{h^{1}(X)}: h \in \overline{H_{0}^{1}}(X)\right\}
$$

in view of (4.2). Suppose that $h \in \overline{H_{0}^{1}}(X)$. Since $S_{n}^{-1} \circ P_{n} \circ h \in \overline{H_{0}^{1}}\left(\ell_{p}^{n}\right)$ and $g_{n}(z) \in M_{n}$ for each $z \in \mathbb{D}$, we get from (6.8) that

$$
\begin{aligned}
n^{1 / p} & \leq\left\|f_{n}\right\|_{C T\left(\ell_{p}^{n}\right)} \leq\left\|f_{n}-S_{n}^{-1} \circ P_{n} \circ h\right\|_{h^{1}\left(\ell_{p}^{n}\right)} \\
& \leq\left\|S_{n}^{-1}\right\| \cdot\left\|g_{n}-P_{n} \circ h\right\|_{h^{1}\left(M_{n}\right)} \leq C\left\|P_{n}\right\| \cdot\left\|g_{n}-h\right\|_{h^{1}(X)} .
\end{aligned}
$$

Hence $\left\|g_{n}\right\|_{C T(X)} \geq C^{-2} n^{1 / p}$ follows from (6.10).

Let $1 \leq p<2$. By using instead the estimate (6.7) and arguing as above we obtain polynomials $g_{n}: D \rightarrow M_{n}$ so that

$$
\left\|g_{n}\right\|_{w C T(X)} \leq n^{1 / s}, \quad\left\|g_{n}\right\|_{C T(X)} \geq C^{-2} n^{1 / p}, n \in \mathbb{N},
$$

where $\frac{1}{s}=\frac{1}{p}-\frac{1}{2}$. Since $\frac{1}{s}<\frac{1}{p}$ the normalized polynomials $h_{n}=n^{-1 / s} g_{n}$ satisfy the requirements for $n \in \mathbb{N}$.

If $X$ is $\mathrm{K}$-convex, then the assumptions are satisfied for $p=2$ by the result cited above. 
Remarks. (i) If $X$ contains a complemented copy of $\ell^{p}$ for some $1 \leq p<\infty$, then one obtains concrete maps $g \in w C T(X) \backslash C T(X)$ by starting from Example 6.4 and suitably modifying the argument of Example 6.2. The technical details are left to the reader.

(ii) One is tempted to conjecture that Example 6.4 should hold for any infinite-dimensional $X$. Unfortunately, the argument of Example 6.4 does not even apply to the case $X=\ell^{\infty}$. The reason is that there is a constant $c>0$ so that $\left\|P_{n}\right\| \geq c \sqrt{n}$ for $n \in \mathbb{N}$ whenever $M \subset \ell^{\infty}$ is 2-isomorphic to $\ell_{2}^{n}$ and $P_{n}$ is a projection of $\ell^{\infty}$ onto $M$, see [Ru, Thm. II and p. 245]. Recall also that Pisier has constructed a Banach space $X$ having the following property: there is a constant $c>0$ so that $\|P\| \geq c \cdot \sqrt{\operatorname{dim}(M)}$ for any finite-dimensional subspaces $M \subset X$ and linear projections $P: X \rightarrow M$ (see $\left[\mathrm{Pi}\right.$, Section 10]). Roughly speaking, the obstruction above is the term $\left\|P_{n}\right\|$ in estimates such as (6.11).

\section{REFERENCES}

[B1] O. Blasco, Boundary values of vector-valued harmonic functions considered as operators, Studia Math. 86: 19-33, 1987.

[B2] O. Blasco, Boundary values of vector-valued functions in Orlicz-Hardy classes, Arch. Math. (Basel) 49: 434-439, 1987.

[B3] O. Blasco, Boundary values of functions in vector-valued Hardy spaces and geometry on Banach spaces, J. Funct. Anal. 78: 346-364, 1988.

[BDL] J. Bonet, P. Domanski, M. Lindström, Weakly compact composition operators on analytic vector-valued function spaces, Ann. Acad. Sci. Fenn. Math. 26: 233-248, 2001.

[BF] J. Bonet and M. Friz, Weakly compact composition operators on locally convex spaces, Math. Nachr. 245: 26-44, 2004.

[BC] P. Bourdon and J.A. Cima, On integrals of Cauchy-Stieltjes type, Houston J. Math. 14: 465-474, 1988.

[Bu] A.V. Bukhvalov, Hardy spaces of vector-valued functions, J. Soviet Math. 16: 1051-1059, 1981.

[BD] A.V. Bukhvalov and A.A. Danilevich, Boundary properties of analytic and harmonic functions with values in Banach spaces (in Russian), Math. Zametki 31: 203-214, 1982. [English translation in Math. Notes 31: 104-110, 1982.]

[CM1] J.A. Cima and A.L. Matheson, Essential norms of composition operators and Aleksandrov measures, Pacific J. Math. 179: 59-64, 1997.

[CM2] J.A. Cima and A. Matheson, Cauchy transforms and composition operators, Illinois J. Math. 42: 58-69, 1998.

[CMR] J.A. Cima, A. Matheson and W.T. Ross, The Cauchy transform. In Quadrature Domains and their Applications, Operator Theory: Advances and Applications vol. 156: 79-111. (Birkhäuser Verlag, 2005)

[C] J.B. Conway, Functions of one complex variable II. (Springer-Verlag, 1995)

[CoM] C.C. Cowen and B.D. MacCluer, Composition operators on spaces of analytic functions. (CRC Press, 1995)

[DU] J. Diestel and J.J. Uhl, jr., Vector Measures. Mathematical Surveys vol. 15. (American Mathematical Society, 1977)

[DJT] J. Diestel, H. Jarchow and A. Tonge, Absolutely summing operators. Cambridge studies in advanced mathematics vol. 43 (Cambridge University Press, 1995).

[DG] S.J. Dilworth and M. Girardi, Bochner vs. Pettis norm: examples and results, Contemp. Math. 144: 69-80, 1993.

[DS] N. Dunford and J.T. Schwartz, Linear operators. Part I. General Theory. (Interscience, 1958)

[D] P.L. Duren, Theory of $H^{p}$ spaces. (Academic Press, 1970) 
[FGR1] F.J. Freniche, J.C. Garcia-Vazquez and L. Rodriguez-Piazza, The failure of Fatou's theorem on Poisson integrals of Pettis integrable functions, J. Funct. Anal. 160: 28-41, 1998.

[FGR2] F.J. Freniche, J.C. Garcia-Vazquez and L. Rodriguez-Piazza, Operators into Hardy-spaces and analytic Pettis integrable functions, In Recent Progress in Functional Analysis, North-Holland Mathematical Studies vol. 189: 349-362. (NorthHolland, 2001)

[H1] W. Hensgen, Hardy-Räume vektorwertiger Funktionen. Dissertation (LudwigMaximilians-Universität Munich, 1986).

[H2] W. Hensgen, On the dual space of $H^{p}(X), 1<p<\infty$, J. Funct. Anal. 92: 348-371, 1990.

[H3] W. Hensgen, Some remarks on boundary values of vector-valued harmonic and analytic functions, Arch. Math. (Basel) 57: 88-96, 1991.

[H4] W. Hensgen, A simple proof of Singer's representation theorem, Proc. Amer. Math. Soc. 124: 3211-3212, 1996.

[HJ] W.E. Hornor and J.E. Jamison, Isometrically equivalent composition operators on spaces of analytic vector-valued functions, Glasgow Math. J. 41: 441-451, 1999.

[L] J. Laitila, Weakly compact composition operators on vector-valued BMOA, J. Math. Anal. Appl. (to appear).

[LT] J. Lindenstrauss and L. Tzafriri, Classical Banach spaces I. Sequence spaces. (Springer-Verlag, 1977)

[LS] M. Lindström and G. Schlüchtermann, Composition of operator ideals, Math. Scand. 84: 284-296, 1999.

[LST] P. Liu, E. Saksman and H.-O. Tylli, Small composition operators on spaces of vector-valued analytic functions, Pacific J. Math. 184: 295-309, 1998.

[N] K. Ng, On a theorem of Dixmier, Math. Scand. 29: 279-180, 1971.

[P] A. Pietsch, Operator ideals. (North-Holland, 1980)

[Pi] G. Pisier, Factorization of linear operators and geometry of Banach spaces, CBMS vol. 60. (American Mathematical Society, 1986)

[R] W. Rudin, Real and complex analysis, 3rd ed. (McGraw-Hill, 1987).

[Ru] D. Rutovitz, Some parameters associated with finite-dimensional Banach spaces, J. London Math. Soc. 40: 241-255, 1965.

[ST] E. Saksman and H.-O. Tylli, Weak compactness of multiplication operators on spaces of bounded linear operators, Math. Scand. 70: 91-111, 1992.

[S1] D. Sarason, Composition operators as integral operators. In Analysis and partial differential equations, pp. 545-565. (Marcel Dekker, 1990)

[S2] D. Sarason, Weak compactness of holomorphic composition operators on $H^{1}$. In Functional analysis and operator theory (New Delhi, 1990), Lecture Notes in Mathematics vol. 1511: 75-79. (Springer-Verlag, 1992)

[Sh1] J.H. Shapiro, The essential norm of a composition operator, Ann. Math. 125: 375-404, 1987.

[Sh2] J.H. Shapiro, Composition operators and classical function theory. (SpringerVerlag, 1993)

[ShS] J.H. Shapiro and C. Sundberg, Compact composition operators on $L^{1}$, Proc. Amer. Math. Soc. 108: 443-449, 1990.

[SB] S.D. Sharma and U. Bhanu, Composition operators on vector-valued Hardy spaces, Extracta Math. 14: 31-39, 1999.

[Si] I. Singer, Sur les applications linéaires intégrales des espaces des fonctions continues I, Rev. Roum. Math. Pures Appl. 4: 391-401, 1959.

[Z] A. Zygmund, Trigonometric series, vol. I and II. 2nd ed. (Cambridge University Press, 1988).

Department of Mathematics and Statistics, P.B. 68 (Gustaf Hällströmin katu 2B), Fin-00014 University of Helsinki, Finland

E-mail address: jussi.laitila@helsinki.fi, hojtylli@cc.helsinki.fi 